\title{
Dynamics of electron emission in double photoionization processes near the Krypton 3d threshold
}

F Penent $\dagger$, S Sheinerman $\nmid \S$, L Andric $\dagger$, P Lablanquie $\dagger$, J

Palaudoux $\dagger$, U Becker $\ddagger$, M Braune $\$$, J Viefhaus $\$$, and J H D Eland $\uparrow$

†LCP-MR, Université Pierre et Marie Curie, 11, rue P et M Curie, 75231 Paris, France

$\S$ Department of Physics, St. Petersburg State Maritime Technical University, 198262

St. Petersburg, Russia

$\ddagger$ Fritz-Haber-Institut der Max-Planck-Gesellschaft, Faradayweg 4-6, 14195 Berlin, Germany

ФPTCL, Oxford University, Oxford OX1 3QZ, United Kingdom

PACS numbers: $32.70 . \mathrm{Jz}, 32.80 . \mathrm{Hd}$

\begin{abstract}
.
Two electron emission following photoabsorption near the $\mathrm{Kr} 3 \mathrm{~d}$ threshold is investigated both experimentally and theoretically. On the experimental side, electron / electron coincidences using a magnetic bottle time of flight spectrometer allow us to observe the complete Double Photo Ionisation (DPI) continua of selected $K r^{2+}$ final states, and to see how these continua are affected by resonant processes in the vicinity of the Kr 3d threshold. The analysis is based on a quantum mechanical approach that takes into account the contribution of three different processes: A) Auger decay of the inner $3 \mathrm{~d}$ vacancy with the associated post collision interaction (PCI) effects, B) capture of slow photoelectrons into discrete states followed by valence multiplet decay (VMD) of the excited ionic states and C) valence shell DPI. The dominant process for each $K r^{2+}\left(4 p^{-2}\right)$ final state is the photoionization of the inner shell followed by Auger decay of the $3 d$ vacancies. Moreover, for the $4 p^{-2}\left({ }^{3} P\right)$ and $4 p^{-2}\left({ }^{1} D\right)$ final ionic states an important contribution comes from the processes of slow photoelectron capture followed by VMD as well as from double ionization of the outer shell involving also VMD.
\end{abstract}




\section{Introduction}

Photon absorption by an atom can lead to the emission of two electrons through different mechanisms, depending on the conditions considered. For example, if the photon energy exceeds slightly the double photoionization (DPI) threshold, then the two emitted electrons have a small energy and we deal with the direct double ionization of the outer shell. When the photon energy is above an inner shell threshold the double ionization is associated usually with inner shell photoionization followed by emission of an Auger electron. Such a kind of DPI is referred to as indirect DPI (see, e.g., the reviews of Briggs and Schmidt 2000, Avaldi and Huetz 2005). Depending on the photon energy and on the final states of the residual ion, many different indirect processes can occur, and it is needed to develop the proper tools for disentangling all the different pathways involved in the electron emission.

One point of primary importance is to define precisely the final ionic state which is created after the Auger decay or after DPI in order to separate the different processes at play and to study the role of electron correlation for each final state. Recently, progress in electron-electron coincidence techniques (Penent et al 2005a) allowed us to achieve such a goal. The first investigations used threshold electron - Auger electron coincidences (Hikosaka et al 2000). They could, for instance, clarify the dynamics of the threshold electron production in the DPI processes near Ar 2p (Lablanquie et al 2005) and Xe 4d inner shell thresholds (Sheinerman et al 2006). A much more powerful coincidence method using a magnetic bottle time of flight spectrometer is able of revealing the complete state selected DPI continua over a much wider range of kinetic energies (Eland et al 2003, Penent et al 2005).

In this paper we have used this last method to measure the evolution with photon energy of state selected $K r^{2+}$ DPI continua in the vicinity of the $3 \mathrm{~d}$ threshold. While the complete DPI continua can be observed, we will concentrate on the edge of these distributions, corresponding to electrons of less than typically $5 \mathrm{eV}$. This choice comes from the fact that most dynamical effects are observed there. It also includes the situation where the $3 \mathrm{~d}$ photoelectron escapes with low kinetic energy. Furthermore it corresponds to the zone where this time of flight technique gives the best experimental energy resolution.

Interpretation and analysis of the experimental spectra are made within the framework of a quantum mechanical model that is fully described in a previous paper (Sheinerman et al 2007). This model has been developed to take into account both the photoionization of inner and outer shells and to describe two kinds of electron correlation which are the most important in the considered energy region, namely post collision interaction (PCI) and valence multiplet decay (VMD). The PCI is known to play a crucial role in resonance processes with slow electron production (see for example the reviews of Kuchiev and Sheinerman 1989, Schmidt 1992). This form of electron correlation involves interaction between the photoelectron and the Auger electron as well as the influence of the ionic field, which varies in the course of the Auger decay, 
on the photoelectron. For small energies of the photoelectron, the PCI distortion leads to a decrease of the photoelectron energy and subsequently to an increase of the Auger electron one. If the exchange of energy between the photoelectron and Auger electron exceeds the excess of the photon energy above threshold, the slow photoelectron can even be captured into a discrete state of the residual ion.

The valence multiplet decay represents the decay by autoionization of a discrete state $n^{\prime} l^{\prime}$ created in the field of the doubly charged ion of $4 p^{-2}\left({ }^{2 S_{1}+1} L_{1}\right)$ term. If this state lies higher than the double ionization threshold of another final ionic state, $4 p^{-2}\left({ }^{2 S+1} L\right)$, then autoionization can occur with emission of a slow autoionizing electron: $4 p^{-2}\left({ }^{2 S_{1}+1} L_{1}\right) n^{\prime} l^{\prime} \rightarrow 4 p^{-2}\left({ }^{2 S+1} L\right)+e_{\text {auto }}$. Initially, the VMD effects were investigated in the decay rates of valence shake-up states of Ne and Ar (Becker et al 1989, Armen and Larkins 1991, 1992, Becker et al 1993). An important role of VMD has been revealed in the threshold electron spectra (Lablanquie et al 2005, Sheinerman et al 2006) and in the population of the highly excited Rydberg states (De Fanis et al 2004, Kitajima et al 2006).

We have carried out a comparison between the experimental and calculated twoelectron emission for the $4 p^{-2}\left({ }^{1} S\right), 4 p^{-2}\left({ }^{1} D\right)$ and $4 p^{-2}\left({ }^{3} P\right) K r^{2+}$ final ionic states. Our analysis shows that three different processes contribute to the DPI yield. One is the PCI distorted Auger decay following creation of a 3d hole, the second is Auger decay where a slow photoelectron is temporarily captured into a discrete quasistationary state and is subsequently re-emitted via VMD and the third is indirect valence double photoionization via creation and decay of intermediate discrete states of the singly charged ion.

The paper is organized as follows. In Section 2, the processes investigated are presented and discussed. In Section 3 the experimental set up is presented and a first analysis of the measured spectra is done. Section 4 presents the description of the theoretical model. In Section 5 the results of calculation and the comparison between the calculated and measured spectra are presented as well as the analysis of the results obtained. The atomic system of units $|e|=m_{e}=\hbar=1$ is used throughout.

\section{Processes}

We consider the DPI process where the residual $K r^{2+}$ ion is left in a precisely defined final state $4 p^{-2}\left({ }^{2 S+1} L\right)$ with $L$ and $S$ being the total angular momentum and spin of the ion. Moreover, we study the case where the photon energy is close to the threshold of the inner shell and one of the emitted electrons has a small energy, in the limits of a few $\mathrm{eV}$. A general classification of the processes which can occur in this case was given in the paper of Sheinerman et al (2007). Here, we specify the processes which can lead to the emission of two electrons near the Kr 3d-threshold. They are schematically represented in Fig 1 as a guide and consist in:

A. The process of $3 d$-subshell ionization with subsequent Auger decay $3 d^{-1} \rightarrow$ 
$4 p^{-2}+e_{\text {Auger }}$. It can be represented in the form:

$$
\begin{aligned}
\gamma+\mathrm{Kr} & \rightarrow \mathrm{Kr}^{+*}\left(3 d^{-1}\right)+e_{\text {photo }}(\tilde{\mathbf{k}}) \rightarrow \\
& \rightarrow \mathrm{Kr}^{2+}\left(4 p^{-2}\left({ }^{2 S+1} L\right)\right)+e_{\text {Auger }}(\mathbf{p})+e_{\text {photo }}(\mathbf{k})
\end{aligned}
$$

The energy $E_{\tilde{k}}$ of the slow photoelectron in the intermediate state differs from its value $E_{k}$ in the final state. This difference is due to the PCI effects: it leads to a decrease of the photoelectron energy.

B. Photoionization of the inner $3 d$-subshell followed by Auger decay and recapture of the slow photoelectron into a discrete state $4 p^{-2}\left({ }^{2 S_{1}+1} L_{1}\right) n^{\prime} l^{\prime}$. As discussed in the introduction, VMD can occur if this discrete state is embedded in a double ionization continuum: $E\left(4 p^{-2}\left({ }^{2 S_{1}+1} L_{1}\right) n^{\prime} l^{\prime}\right)>E\left(4 p^{-2}\left({ }^{2 S+1} L\right)\right)$. Autoionizing electrons are emitted with energies $0<E_{k}<4.1 \mathrm{eV}$ because the difference of the doubly charged ionic states $\mathrm{Kr}^{2+}\left(4 p^{-2}\right)$ with different terms lies within this limit. The process of the PCI capture of the photoelectron in an intermediate state followed by VMD can be represented as

$$
\begin{aligned}
& \gamma+\mathrm{Kr} \rightarrow \mathrm{Kr}^{+}\left(3 d^{-1}\right)+e_{\text {photo }}(\tilde{\mathbf{k}}) \rightarrow \mathrm{Kr}^{2+}\left(4 p^{-2}\left({ }^{2 S_{1}+1} L_{1}\right)\right)+e_{\text {photo }}(\tilde{\mathbf{k}})+e_{\text {Auger }}(\tilde{\mathbf{p}}) \rightarrow \\
& \rightarrow \mathrm{Kr}^{+*}\left(4 p^{-2}\left({ }^{2 S_{1}+1} L_{1}\right) n^{\prime} l^{\prime}\right)+e_{\text {Auger }}(\mathbf{p}) \rightarrow \mathrm{Kr}^{2+}\left(4 p^{-2}\left({ }^{2 S+1} L\right)\right)+e_{\text {Auger }}(\mathbf{p})+e_{\text {auto }}(\mathbf{k}),
\end{aligned}
$$

where $L_{1}, S_{1}$ are the angular momentum and spin of the ionic core in the intermediate state.

Note that if the photon energy is lower than the inner shell threshold then no photoelectron can be created in the continuum . But, in this case, the population of the $\mathrm{Kr}^{+*}\left(4 p^{-2}\left({ }^{2 S_{1}+1} L_{1}\right) n^{\prime} l^{\prime}\right)$ state may occur through a resonant Auger decay (Armen et al 2000). In our case this decay has the form $\operatorname{Kr}^{*}\left(3 d^{-1} n_{1} l_{1}\right) \rightarrow \mathrm{Kr}^{+*}\left(4 p^{-2}\left({ }^{2 S_{1}+1} L_{1}\right) n^{\prime} l^{\prime}\right)+$ $e_{\text {Auger }}(\mathbf{p})$. The total scheme of this process is written as

$$
\begin{aligned}
\gamma+\mathrm{Kr} & \rightarrow \mathrm{Kr}^{*}\left(3 d^{-1} n_{1} l_{1}\right) \rightarrow \mathrm{Kr}^{+*}\left(4 p^{-2}\left({ }^{2 S_{1}+1} L_{1}\right) n^{\prime} l^{\prime}\right)+e_{\text {Auger }}(\mathbf{p}) \rightarrow \\
& \rightarrow \mathrm{Kr}^{2+}\left(4 p^{-2}\left({ }^{2 S+1} L\right)\right)+e_{\text {Auger }}(\mathbf{p})+e_{\text {auto }}(\mathbf{k}) .
\end{aligned}
$$

C. Photoionization of the outer $4 p$ shell with excitation of another $4 p$ electron into a discrete orbital. As well as in process (2) the resulting intermediate excited state can decay by VMD if it lies above a final doubly charged ionic state. This scheme of double ionization of the outer shell including the VMD processes can be written in the form:

$$
\begin{aligned}
\gamma+\mathrm{Kr} & \rightarrow \mathrm{Kr}^{+*}\left(4 p^{-2}\left({ }^{2 S_{1}+1} L_{1}\right) n^{\prime} l^{\prime}\right)+e_{\text {photo }}(\mathbf{p}) \rightarrow \\
& \rightarrow \mathrm{Kr}^{2+}\left(4 p^{-2}\left({ }^{2 S+1} L\right)\right)+e_{\text {photo }}(\mathbf{p})+e_{\text {auto }}(\mathbf{k})
\end{aligned}
$$

There is also another process for the valence electrons ionization. It is the direct double ionization of the outer $4 p$-shell:

$$
\gamma+\mathrm{Kr} \rightarrow \mathrm{Kr}^{2+}\left(4 p^{-2}\left({ }^{2 S+1} L\right)\right)+e_{1}(\mathbf{k})+e_{2}(\mathbf{p}) .
$$

The direct DPI of the outer shell has the following characteristics (Briggs and Schmidt 2000, Avaldi and Huetz 2005). i) The cross section of the process depends smoothly on 
the photon energy. ii) The process goes on without creation and decay of intermediate resonant state and the electron distribution is monotonic. iii) Cross section for the direct process (5) is much less than the cross sections of the resonant processes (1)-(4). Hence the direct double ionization of the outer shell represents only a small part of the total cross section for two electron emission. This is why we will neglect the direct double photoionization of the outer $4 p$ shell in our analysis and limit ourselves to processes (1)-(4) only.

\section{Coincidence measurements for two electron emission}

\subsection{Experimental set-up}

The experiment was performed at BESSY on beam line UE56/2-PGM-1, during single bunch operation of the storage ring, which provides light pulses of a few tens of ps width every $800.5 \mathrm{~ns}$. The photon resolution was of the order of a few meV. The core of our HERMES (High Energy Resolution Multi Electron Spectroscopy) experimental set-up is a magnetic bottle time of flight spectrometer of the type developed by Eland et al (Eland et al 2003). The present apparatus has been described elsewhere (Penent et al 2005, Lablanquie et al 2007). Briefly, a strong permanent magnet (0.5 T) located close to the source volume and a long solenoid $\left(10^{-3} T\right)$ create a magnetic mirror that guides essentially all produced electrons to the detector located at the end of the 2.4 $\mathrm{m}$ flight tube. The position of the magnet was adjusted with a XYZ manipulator to optimize the time of flight resolution and the image of the collision center on the detector (visualized directly on a phosphor screen). A repelling potential $(\sim 0.5 \mathrm{~V})$ was applied to the magnet to accelerate low energy electrons so that they arrive to the detector in a finite time (less than $6 \mu \mathrm{s}$ here). A multi-hit time-to-digital converter (TDC) with 250 ps resolution was used to reference the electron times of flight with respect to the light pulses and to record electron-electron coincidences. Calibration for the conversion from the electron time of flight to energy was performed by measuring He photoelectron spectra at known photon energies. The electron count rate was maintained at around $10 \mathrm{kHz}$ by adjusting the photon flux, in order to keep a reasonable amount of random coincidences.

\subsection{Presentation of the experimental data}

Two-dimensional electron-electron coincidence maps have been measured at selected energies around Kr 3d thresholds. An example is chosen in Fig 2 (top), which contains all features present in other spectra. It was measured at a photon energy of $95.24 \mathrm{eV}$, that is $200 \mathrm{meV}$ above the $\mathrm{Kr} 3 d_{3 / 2}$ threshold. Diagonal lines of constant kinetic energy sum trace the formation of the different $K r^{2+}$ final states. The $K r^{2+} 4 p^{-2}\left({ }^{3} P\right)$ state is partly resolved into its ${ }^{3} P_{2}$ and ${ }^{3} P_{0,1}$ components. The intensity along the lines show the energy sharing between the 2 electrons for the different DPI continua. Three of them, corresponding to the formation of the $K r^{2+} 4 p^{-2}$ levels are represented in Fig 2 
(bottom). Only the low energy part of the DPI continua is represented because the high energy part is its mirror image, but is affected by a worse experimental resolution.

In order to understand better the different contributions, we have selected in Fig 3 two final states, namely $K r^{2+}\left(4 p^{-2}\right){ }^{3} P_{2}$ (bottom curves) and ${ }^{1} D$ (top curves), and compared the associated DPI continua populated at $95.24 \mathrm{eV}$ photon energy Fig 3 (right panel) with the ones obtained at two other photon energies. Fig 3 (left panel) shows the spectra obtained at $85 \mathrm{eV}$, below the $3 \mathrm{~d}$ excitation region. Valence DPI (process $\mathrm{C}$ in section 2 ) is then the only possible contribution. The ${ }^{1} D$ DPI continuum is populated by the direct valence DPI (equation (5)), which manifests itself by a weak U-shape continuous intensity. The ${ }^{3} P_{2}$ DPI continuum, on the other hand contains only weak contributions of direct valence DPI and is dominated by indirect processes (equation (4)), as evidenced by the peaked structure between 0 and $2 \mathrm{eV}$. This structure corresponds to the VMD of Rydberg series converging to the $K r^{2+}\left(4 p^{-2}\right){ }^{1} D$ state. Comparison with the photoelectron spectra in (Alitalo et al 2001) suggests a dominant series of $K r^{*+}\left(4 p^{-2}\right)\left({ }^{1} D\right) n d\left({ }^{2} S\right)$ configuration. The validity of such an assignment was discussed in Eland et al (2003) for the equivalent xenon case. Note that the VMD $4 p^{-2}\left({ }^{1} D\right) n d\left({ }^{2} S\right) \rightarrow 4 p^{-2}\left({ }^{3} P\right)+e_{\text {auto }}(\mathbf{k})$ is forbidden due to the parity conservation law. However in our case this transition occurs in the presence of the photoelectron $e_{\text {photo }}(\mathbf{p})$ (see the eq.(4)). Hence exchange of angular momentum between the electrons $e_{\text {photo }}(\mathbf{p})$ and $e_{\text {auto }}(\mathbf{k})$ can probably occur and VMD of the $4 p^{-2}\left({ }^{1} D\right) n d\left({ }^{2} S\right)$ state takes place leading to autoionization. Other series seem to be important at lower photon energies as observed in (Eland et al 2003). The electron energy cut-off associated with high $\mathrm{n}$ Rydbergs occurs at the difference of binding energy between the $\mathrm{Kr}^{2+}\left(4 p^{-2}\right)$ ${ }^{1} D$ and ${ }^{3} P_{2}$ states at $1.816 \mathrm{eV}$ (NIST 2007). We deal in fact here with the population of the $K r^{2+}\left(4 p^{-2}\right)^{1} D$ channel: the continuum part is observed in the $K r^{2+}{ }^{1} D$ DPI continuum while the discrete part is observed through its autoionization into the $K r^{2+}$ ${ }^{3} P_{2}$ continuum. Autoionization to the $K r^{2+}{ }^{3} P_{1,0}$ channels is found to be about 3 times less intense, which explains the similar count rates at the $0 \mathrm{eV}$ edge of the $K r^{2+}\left(4 p^{-2}\right)$ ${ }^{1} D$ DPI continuum and at the cut off mentioned above (continuity in the $K r^{2+}{ }^{1} D$ DPI channel). The spectra of the central pannel in Fig 3 have been obtained at 98.54 $\mathrm{eV}$ photon energy, $3.5 \mathrm{eV}$ above the $3 d_{3 / 2}$ threshold. The same valence DPI processes as above are present, but the dominant contribution to the electron signal is now due to the Auger decay of the $3 \mathrm{~d}$ holes (process A in section 2). A PCI distortion of the coincidence peaks is clearly visible.

At $95.24 \mathrm{eV}$ photon energy (Fig 3, right panel) the capture of the slower $3 d_{3 / 2}$ photoelectron followed by its re-emission by VMD takes place (equation $(2)$ ). The ${ }^{1} D$ DPI continuum shows that the $3 d_{5 / 2}$ photoelectron peak has moved down to $1.35 \mathrm{eV}$ and that only a part of the distorted $3 d_{3 / 2}$ photoelectron peak is observed above $0 \mathrm{eV}$. The missing part, expected for un-physical negative electron energies, corresponds in fact to the capture of the $3 d_{3 / 2}$ photoelectrons in $K r^{*+}\left(4 p^{-2}\right)\left({ }^{1} D\right) n l$ Rydberg states with simultaneous emission of a fast Auger electron. The subsequent autoionization of these Rydberg states by VMD is observed here in the ${ }^{3} P_{2}$ DPI continuum. Note that 
capture is preferentially into high levels with $n>10$. In the same way, the Rydberg structure observed in the ${ }^{1} D$ DPI continuum (Fig 3 top curve, right panel) corresponds to the capture of the $3 d_{3 / 2}$ photoelectron in the $\left(4 p^{-2}\right)\left({ }^{1} S\right)$ channel. The Rydberg series involved in the capture are predominantly $\left(4 p^{-2}\right)\left({ }^{1} S\right) n p$ and $\left(4 p^{-2}\right)\left({ }^{1} D\right) n p$, as was established by Aksela et al (1997), and indeed we observe similar quantum defects for the two dominant Rydberg series. Surprisingly, the position of the $K r^{*+}\left(4 p^{-2}\right)\left({ }^{1} D\right) n p$ Rydberg states to which recapture occurs is found to coincide, within our experimental resolution, with that of the $K r^{*+}\left(4 p^{-2}\right)\left({ }^{1} D\right)(n-1) d\left({ }^{2} S\right)$ states populated by indirect valence double ionization at $85 \mathrm{eV}$ (equation (4)).

Back to the coincidence map in Fig 2 (top panel), the $3 d_{5 / 2}$ photoelectron / Auger electron coincidences give rise to the spots on an horizontal line with a $1.35 \mathrm{eV}$ energy ordinate. Most of the other features reveal the PCI capture of the $3 d_{3 / 2}$ photoelectron into the different $K r^{2+}$ channels, followed by their autoionization by VMD to lower $K r^{2+}$ states. For instance capture into the $K r^{2+}\left({ }^{1} S\right)$ channel form intermediate $K r^{*+}\left(4 p^{-2}\right)\left({ }^{1} S\right) n l$ states with the simultaneous emission a fast Auger electron of around $53 \mathrm{eV}$. Subsequent autoionization by VMD of these intermediate states to the lower $K r^{2+}\left({ }^{1} D\right)$ and $\left({ }^{3} P\right)$ states are revealed by the spots with an abscissa around $53 \mathrm{eV}$ and appear on the diagonal lines corresponding to these final states. Similar considerations allow us to identify all the capture processes of the $3 d_{3 / 2}$ photoelectron into the different $K r^{2+}$ channels, as reported in Fig 2. As an example, the spots associated with a fast electron of around $39 \mathrm{eV}$ indicate the capture of the $3 d_{3 / 2}$ photoelectron into the $K r^{2+}$ $4 s^{-1} 4 p^{-1}\left({ }^{1} P\right)$ channel. Similar processes were identified for Ar $2 \mathrm{p}$ by Feng et al in their non coincidence experiment (Feng et al 2005). Finally the autoionization of highly excited $K r^{+*}$ states imbedded in the DPI continuum (Kikas et al 1996) is responsible for the weak structure in the $4 p^{-2}{ }^{1} \mathrm{D}$ and ${ }^{3} \mathrm{P}$ continua for fast electrons of $45-50 \mathrm{eV}$ energies.

In the following we will focus on the low energy part of the spectrum (electron energies of less than $5 \mathrm{eV}$ ), and the above assignments will be validated by our calculations.

\section{Calculation for two electron emission}

\subsection{Theoretical model}

In order to calculate the amplitudes of different resonant processes for two electron emission an unified approach was developed (Sheinerman et al 2007) in the framework of quantum mechanical many-body theory. We will apply this approach to the processes (1)-(4) when the two electrons with momenta $\mathbf{k}, \mathbf{p}$ are ejected and the residual ion is left in the state $4 p^{-2}\left({ }^{2 S+1} L\right)$. Due to the energy conservation

$$
\omega=E_{k}+E_{p}-E\left(\operatorname{Kr}^{2+}\left(4 p^{-2}\left({ }^{2 S+1} L\right)\right)\right)
$$

the coincidence measurements of the energies $E_{k}$ and $E_{p}$ define precisely the final ionic state for every selected energy $\omega$ of incident photon. The amplitudes of processes are 
marked as $A_{\text {inner }}^{L M}(\omega, \mathbf{k}, \mathbf{p})$ for the process $(1), A_{\text {inner-val }}^{L M S M_{S}}(\omega, \mathbf{k}, \mathbf{p})$ for the processes $(2)$ and (3) (note that both the processes (2) and (3) are considered as processes of the same kind), and $A_{\text {valence }}^{L M S M_{S}}(\omega, \mathbf{k}, \mathbf{p})$ for the process (4), where $M$ and $M_{S}$ are the projections of the angular momentum and spin of the final ionic state. The general expressions for these amplitudes are given in the paper of Sheinerman et al (2007). A short description and physical explanation of the model used are presented below.

Calculating the amplitude $A_{\text {inner }}^{L M}(\omega, \mathbf{k}, \mathbf{p})$ for the process (1) we have first of all to take into account the PCI effects. We study the near-threshold region. Hence, the energy of the photoelectron can be considered much smaller than the energy of the Auger electron $\left(E_{\text {photo }} \lesssim 5 \mathrm{eV}, E_{\text {Auger }} \sim 40-50 \mathrm{eV}\right)$. The fast Auger electron rapidly leaves the zone of reaction and the direct interaction between the photoelectron and the Auger electron can be neglected. In this case, the PCI reduces to the interaction between the slow photoelectron and the field of the ion target which varies in the course of Auger decay. The amplitude of such a process can be presented through the overlap integral (Kuchiev and Sheinerman 1985, 1986)

$$
\left\langle P_{E_{k}, l}|| a_{\omega, l}\right\rangle=\int_{0}^{\infty} P_{E_{k}, l}^{*}(r) a_{\omega, l}(r) d r .
$$

Here $P_{E_{k}, l}$ is the radial part of the final photoelectron wave function calculated in the field of the doubly charged ion. The function $a_{\omega l}(r)$ is the radial part of the function $A(\omega, \mathbf{r})$, which describes the creation of the photoelectron and its propagation to the point $\mathbf{r}$ in the field of the singly charged ion. This function is found as solution of the inhomogeneous differential equation (see Kuchiev and Sheinerman 1985, Sheinerman 2003). Solving this equation we obtain the functions $a_{\omega l}(r)$ which are used for the calculation of the amplitude. Both the possible transitions $3 d \rightarrow E_{k} p$ and $3 d \rightarrow E_{k} f$ are taken into account in the amplitude. Hence it is represented as a linear combinations of the overlap integrals $\left\langle P_{E_{k}, p} \| a_{\omega, p}\right\rangle$ and $\left\langle P_{E_{k}, f} \| a_{\omega, f}\right\rangle$.

Besides the overlap integrals, the amplitude $A_{\text {inner }}^{L M}(\omega, \mathbf{k}, \mathbf{p})$ is determined by the matrix element of the Auger decay. Note that our calculation is carried out in the LScoupling scheme with one-electron wave functions calculated in the Hartree-Fock (HF) approximation. Then the two electron wave function of the final ionic state is written as:

$$
\Psi_{i o n}^{L M}\left(\mathbf{r}_{a}, \mathbf{r}_{b}\right)=\sum_{m, m^{\prime}} C_{1 m 1 m^{\prime}}^{L M} \varphi_{4 p m}\left(\mathbf{r}_{a}\right) \varphi_{4 p m^{\prime}}\left(\mathbf{r}_{b}\right)
$$

where $C_{1 m 1 m^{\prime}}^{L M}$ is the Clebsch-Gordan coefficient and $\varphi_{4 p m}\left(\mathbf{r}_{i}\right)$ is the one electron wave function of the outer $4 \mathrm{p}$-shell vacancy. In this case the matrix element of the Auger decay can be presented through a multiplication of the $3 \mathrm{j}$-symbols and the reduced matrix element of the Auger decay $\left\langle 3 d, E_{p} l_{p}\left\|V_{g}\right\| 4 p, 4 p\right\rangle$ (its definition can be found, for example, in Amusia 1990). The final expression for the amplitude $A_{\text {inner }}^{L M}(\omega, \mathbf{k}, \mathbf{p})$ is determined by the combination of the overlap integrals (7), reduced matrix elements of the Auger decay and $3 \mathrm{j}$-symbols.

To calculate the amplitude $A_{\text {inner-val }}^{\operatorname{LMSM}_{S}}(\omega, \mathbf{k}, \mathbf{p})$ for the processes (2) and (3), one has to take into account that these processes are formed by two transitions: from the 
initial state to the intermediate state $4 p^{-2}\left({ }^{2 S_{1}+1} L_{1}\right) n^{\prime} l^{\prime}$ and from this intermediate state to the final ionic state $4 p^{-2}\left({ }^{2 S+1} L\right)$ with emission of the autoionizing electron. The first transition is determined by the overlap integral (7) whereas the second transition - by the matrix element of the VMD.

According to the rules of quantum mechanics, all real and virtual excited states of the singly charged ion in a selected channel can form the intermediate state. It means that to evaluate the amplitude of the processes (2), (3) we have to take into account all the possible electron states $n^{\prime} l^{\prime}$ both in the discrete spectrum and the continuum. Then the amplitude can be presented by the integral:

$$
\begin{aligned}
& I\left(\omega, E_{k}, l_{k}, l_{a}, g, M_{s}\right)= \\
& \quad=\int d E_{q} \frac{\left\langle 4 p, E_{k} l_{k} \| \hat{U}_{g}\left(M_{S}\right)|| E_{q} l_{q}, 4 p\right\rangle\left\langle P_{E_{q} l_{q}} \| a_{\omega, l_{a}}\right\rangle}{\omega-E_{p}-E_{q}+E\left(K r^{2+}\left(L_{1}\right)\right)+i \Gamma_{2} / 2} \cdot \delta_{l_{a}, l_{q}},
\end{aligned}
$$

where the overlap integral is given by the eq.(7) and the reduced matrix elements of the VMD $\left\langle\ldots|| \hat{U}_{g}\left(M_{S}\right)|| \ldots\right\rangle$ are expressed through direct and exchange Coulomb matrix elements (Amusia 1990). Note that these matrix elements are calculated also in the HF approximation. The wave functions of the excited electron states $P_{E_{q} l_{q}}(r)$ can belong both to the discrete spectrum and to the continuum in the field of the double charged ion state $K r^{2+}\left(4 p^{-2}\left(L_{1} M_{1}\right)\right)\left(L_{1}, M_{1}\right.$ are the angular momentum of the ionic intermediate state and its projection). Hence the integration over $d E_{q}$ in the eq.(9) includes the sum over all possible states of the discrete spectrum. The energies $E_{p}, E_{q}$ and $E\left(K r^{2+}\left(L_{1}\right)\right)$ are respectively the energies of the electron in the states $|\mathbf{p}\rangle,|\mathbf{q}\rangle$ and of the doubly charged ion $K r^{2+}\left(L_{1} M_{1}\right) . \Gamma_{2}$ is the width of the intermediate excited state.

Finally the amplitude $A_{\text {inner-val }}^{L M S M_{S}}(\omega, \mathbf{k}, \mathbf{p})$ is expressed through the combination of the integrals $I(9)$, the reduced matrix elements of the Auger decay, $3 \mathbf{j}-$ and $6 \mathbf{j}$-symbols.

Now let's concentrate on the amplitude $A_{\text {valence }}^{\operatorname{LMSM}_{S}}(\omega, \mathbf{k}, \mathbf{p})$ for the valence shell DPI, eq.(4). This process occurs also in two steps. In the first step an outer shell electron is ionized with simultaneous excitation of another valence electron into the excited state $n^{\prime} l^{\prime}$. In the second step the VMD of the $K r^{+*}$ ion occurs with ejection of the autoionizing electron. Because an explicit quantum mechanical consideration of the two-step processes demands that we take into account all the possible intermediate electron states $n^{\prime} l^{\prime}$ both of the discrete spectrum and the continuum, the first step of the process (4) should be considered as a general process of double ionization of the outer shell. There are different mechanisms for outer shell direct double photoionization in many-electron atoms: correlations in the initial state, knocking out of second electron by first electron, shake-off (Chang and Poe 1975, Carter and Kelly 1977). For simplicity, our approach is restricted to the easiest shake process. According to this approximation one of the electrons of the valence shell absorbs a photon and is ejected into continuum. The other electron of the valence shell is emitted into continuum or excited into discrete state due to the shake process as it feels a change of the field inside the atom. In this case the keystone of calculation of the amplitude $A_{\text {valence }}^{\operatorname{LMSM}_{S}}(\omega, \mathbf{k}, \mathbf{p})$ presents the integral

$$
I_{1}\left(\omega, E_{p}, E_{k}, l_{k}, g, M_{s}\right)=
$$




$$
=\int d E_{q} \frac{\left\langle 4 p, E_{k} l_{k}\left\|\hat{U}_{g}\left(M_{S}\right)\right\| E_{q} l_{q}, 4 p\right\rangle\left\langle E_{q} l_{q} \| 4 p\right\rangle \delta_{l_{q}, 1}}{\omega-E_{p}-E_{q}+E\left(K r^{2+}\left(L_{1}\right)\right)+i \Gamma_{2} / 2},
$$

where $\left\langle 4 p, E_{k} l_{k}\left\|\hat{U}_{g}\left(M_{S}\right)\right\| E_{q} l_{q}, 4 p\right\rangle$ is the reduced matrix element of the valence multiplet decay; $\left\langle E_{q} l_{q} \| 4 p\right\rangle$ is the reduced matrix element of the shake process:

$$
\left\langle E_{q} l_{q} \| 4 p\right\rangle=\int P_{E_{q} l_{q}}(r) P_{4 p}(r) d r .
$$

The final expression for the amplitude $A_{\text {valence }}^{\operatorname{LMSM}_{S}}(\omega, \mathbf{k}, \mathbf{p})$ is determined by the combination of the integrals $I_{1}$, reduced matrix elements of the photon absorption $\left\langle E_{p} l_{p}\left\|\nabla_{1}\right\| 4 p\right\rangle$ (see, Amusia 1990), $3 \mathbf{j}-$ and $6 \mathbf{j}$-symbols.

The cross section for the two electron emission is given by the square of the modulus of the sum of amplitudes and can be written as

$$
\frac{d \sigma}{d E_{k}}(\omega)=\frac{64 \pi^{2}}{c \omega} \int d \Omega_{p} d \Omega_{k} \sum_{M, M_{S}}\left|\left(A_{\text {inner }}^{L M}+A_{\text {inner-val }}^{L M S M_{S}}+A_{\text {valence }}^{L M S M_{S}}\right)\right|^{2},
$$

where the amplitudes in parenthesis describe the processes (1)-(4). It should be noted that the numerical factor before the integral takes into account that the HF one-electron radial wave functions which are used for calculation of the amplitudes are normalized on the energy scale (in Ry) to a delta-function. Carrying out the integration over the solid angles $\Omega_{k}, \Omega_{p}$ and summation over the magnetic quantum number $M$, we can present the final form of the cross section as a sum of 17 terms:

$$
\frac{d \sigma}{d E_{k}}(\omega)=\frac{64 \pi^{2}}{c \omega} \sum_{M_{S}}\left(\sum_{i=1}^{17} T_{i}\right)
$$

The equations for the terms $T_{1}-T_{17}$ are given in Sheinerman et al (2007). They are expressed through combinations of the $6 \mathrm{j}$-symbols, integrals $I, I_{1}$ (see the eq.(9), (10)) and matrix elements which are presented above. Note that the sum (13) includes interference terms and allows us to calculate coherent and incoherent contribution of different processes to the cross section.

\subsection{Calculation of the cross section}

We consider only the $\mathrm{Kr}^{2+}$ ions with two $4 \mathrm{p}$ vacancies. As our calculations are carried out in the scheme of LS-coupling we consider three different final states of the doubly charged $K r^{2+}\left(4 p^{-2}\right)$ ion: $4 p^{-2}\left({ }^{3} P\right), 4 p^{-2}\left({ }^{1} D\right), 4 p^{-2}\left({ }^{1} S\right)$. The energies of these states calculated in the HF approximation differ approximately by $2 \mathrm{eV}$ from the exact experimental values (Sheinerman et al 2007). Calculating the integrals $I$, $I_{1}$, in eqs.(9), (10) and the overlap integrals (7) we have used the experimental values $E\left({ }^{1} D_{2}\right)=40.175 \mathrm{eV}, E\left({ }^{1} S_{2}\right)=42.461 \mathrm{eV}$ for the $\left({ }^{1} \mathrm{D}\right),\left({ }^{1} \mathrm{~S}\right)$ ionic states and have chosen the averaged experimental value $E=38.76 \mathrm{eV}$ for description of the $4 p^{-2}\left({ }^{3} \mathrm{P}\right)$ state (NIST data).

In the case of the final ionic state $4 p^{-2}\left({ }^{3} P\right)$ there are two intermediate ionic states $4 p^{-2}\left({ }^{1} D\right)$ and $4 p^{-2}\left({ }^{1} S\right)$ which lie higher than the state $4 p^{-2}\left({ }^{3} P\right)$. They can 
contribute to the cross section by means of the processes (2)-(4). Only the intermediate state $4 p^{-2}\left({ }^{1} D\right)$ has been taken into account in this case because the energies of the autoionizing electrons from the VMD of the $4 p^{-2}\left({ }^{1} D\right) n^{\prime} l^{\prime}$ states are located just in the region $0-2 \mathrm{eV}$, where a rich structure was revealed in the measured spectra. In the case of the $4 p^{-2}\left({ }^{1} D\right)$ final ionic state we have taken into account the intermediate $4 p^{-2}\left({ }^{1} S\right)$ state which lies higher than the final state and contributes through processes $(2)-(4)$. For the highest final ionic state $4 p^{-2}\left({ }^{1} S\right)$ only process (1) can contribute to the cross section .

Our calculations were carried out for three different energies of the incident photon: $\omega=100.04 \mathrm{eV}, \omega=95.24 \mathrm{eV}$ and $\omega=94.74 \mathrm{eV}$. These energies correspond respectively to an excess photon energy of $\Delta E=5.0 \mathrm{eV}, 0.2 \mathrm{eV}$ above the $3 d_{3 / 2}$-subshell threshold, and of $\Delta E=-0.3 \mathrm{eV}$ below the $3 d_{3 / 2}$-subshell threshold. The choice of these energies is conditioned by the different role of processes (1)-(4) in two electron emission (see Fig 3). For the first photon energy we expect a very minor role of the PCI capture effects. Hence the cross section has to be determined by processes (1) and (4). For $\omega=95.24 \mathrm{eV}$ the PCI capture is expected to be very important and all three processes (1), (2) and (4) contribute to the cross section. The lower photon energy lies just below the threshold and the resonant Auger decay of reaction (3) occurs. So for this energy the processes (1), (3) and (4) are important.

Carrying out the calculations of the amplitudes $A_{\text {inner }}^{L M}$ and $A_{\text {inner-val }}^{L M S M_{S}}$ we take into account the transition of the $3 \mathrm{~d}$-electron both with the increase and the decrease of the angular momentum: $3 d \rightarrow \varepsilon p, 3 d \rightarrow \varepsilon f$. Apart from this, our calculation takes into account that the intermediate states $4 p^{-2}\left({ }^{2 S_{1}+1} L_{1}\right) \varepsilon p, 4 p^{-2}\left({ }^{2 S_{1}+1} L_{1}\right) \varepsilon f$ can be populated by the transition of the $3 d$-electron from two subshells: $3 d_{3 / 2}$ and $3 d_{5 / 2}$. Because of different ionization energies of these subshells: $E\left(3 d_{5 / 2}\right)=93.79 \mathrm{eV}$, $E\left(3 d_{3 / 2}\right)=95.04 \mathrm{eV}$ (Schmidt 1997) the excess energy above the threshold for the $3 d_{3 / 2}$ electron differs from the $3 d_{5 / 2}$ one for the same energy of the incident photon. As a consequence of this fact the amplitudes $a_{\omega, l}\left(M_{4,5}\right)$ which describe the transitions of the inner electron from the subshells $3 d_{3 / 2}, 3 d_{5 / 2}$ are different. Hence the matrix element (5) is presented as a linear combination of these amplitudes:

$$
\left\langle P_{E_{k}, l} \| a_{\omega, l}\right\rangle=a_{1}\left(M_{4}\right)\left\langle P_{E_{k}, l} \| a_{\omega, l}\left(M_{4}\right)\right\rangle+a_{1}\left(M_{5}\right)\left\langle P_{E_{k}, l} \| a_{\omega, l}\left(M_{5}\right)\right\rangle
$$

where the factors $a_{1}\left(M_{4,5}\right)$ give the relative strength of these transitions. In our calculations these factors were chosen in accordance with the statistical weights of the $3 d_{3 / 2}$ and $3 d_{5 / 2}$ subshells.

The widths of the inner vacancy and intermediate states have to be considered as parameters in the calculation. The widths $\Gamma$ of the $3 d$-holes are equal to $88 \mathrm{meV}$ (Jurvansuu et al 2001). The value of the width $\Gamma_{2}$ of the intermediate excited states $4 p^{-2}\left({ }^{2 S+1} L_{1}\right) n^{\prime} l^{\prime}$ was chosen equal to $20 \mathrm{meV}$ in accordance with the experimental resolution of the coincidence measurements which give that $\Gamma_{2} \leq 20 \mathrm{meV}$ (Eland et al 2003).

Calculation of the amplitude $A_{\text {valence }}^{L M S M_{S}}$ in our model takes into account that one of 
the valence electrons absorbs the photon giving rise to the transitions $4 p \rightarrow \varepsilon s, 4 p \rightarrow \varepsilon d$ whereas the second valence electron takes part in the shake transition $4 p \rightarrow \varepsilon p$. For the calculation of the integrals $I, I_{1}$ in eqs.(9), (10) we take into account the contribution of the discrete spectrum adding the sum over the lowest 11 discrete excited states $n_{q} l_{q}$.

The matrix elements of the VMD $\left\langle 4 p, E_{k} l_{k}\left\|\hat{U}_{g}\left(M_{S}\right)\right\| E_{q} l_{q}, 4 p\right\rangle$ have been calculated in the $\mathrm{HF}$ approximation. In this case the wave functions of hole states $4 p^{-1}$ were found from the calculation of the self-consistent configuration $K r^{+}\left(\ldots 3 d^{10} 4 s^{2} 4 p^{5}\right)$. The electron wave function in the intermediate state $E_{q} l_{q}$ were calculated in the field of $K r^{2+}$ ion with configuration ...3 $3 d^{10} 4 s^{2} 4 p^{4}\left({ }^{2 S_{1}+1} L_{1}\right)$. The final electron wave functions of the state $E_{k} l_{k}$ were calculated in the field of the $K r^{2+}\left(\ldots 3 d^{10} 4 s^{2} 4 p^{4}\left({ }^{2 S+1} L\right)\right)$ configuration. These wave functions are not orthogonal and their use is justified by the physical picture of the processes considered. On the other side a calculation of the matrix elements with the eigenfunctions of the different Hamiltonians corresponds to taking into account some of the many-electron corrections beyond the HF approximation (Amusia 1990). Note that the many-electron correlation influences significantly the VMD rates (Armen and Larkins 1991, 1992). However we restrict ourselves to the easiest calculation of the VMD matrix elements for each of the processes (2)-(4) because the accurate account of the electron correlation in the VMD is quite complicated and lies beyond our approach.

\section{Results and analysis}

\subsection{Results of calculation}

The calculated cross sections of the two electron ejection are presented in Fig 4, 5 and 6 as a function of the energy of the slow emitted electron for three different photon energies $\omega=100.04 \mathrm{eV}$ (Fig 4), $\omega=95.24 \mathrm{eV}$ (Fig 5) and $\omega=94.74 \mathrm{eV}$ (Fig 6). The top panel of each figure is associated with the final ionic state $4 p^{-2}\left({ }^{1} S\right)$, the medium part with the $4 p^{-2}\left({ }^{1} D\right)$ final states and the bottom panel with the $4 p^{-2}\left({ }^{3} P\right)$ states. The solid lines on these figures present the full calculation taking into account the interference terms (see Sheinerman et al 2007) whereas the dashed lines describe the incoherent contribution of the processes (1)-(4) to the cross section. A preliminary analysis of the calculated curves for the final states $4 p^{-2}\left({ }^{1} D\right)$ and $4 p^{-2}\left({ }^{3} P\right)$ was done in our previous publication (Sheinerman et al 2007). Here we present the total results of the calculation.

On Fig 4 we can clearly see two peaks located near $4.9 \mathrm{eV}$ and $6.1 \mathrm{eV}$. These peaks are present for every final ionic states and are associated with emission of a slow photoelectron from the inner $3 d_{3 / 2}$ and $3 d_{5 / 2}$ subshells via the process (1). The line shapes of these peaks are distorted by the PCI: the maxima are shifted towards a low energy (maxima of unshifted distributions are located at 5.0 and $6.25 \mathrm{eV}$ ) and the peaks have an asymmetrical form. The relative strength of these peaks is determined by the matrix elements of the Auger decay and the statistical weights of the $3 d_{3 / 2}$ and $3 d_{5 / 2}$ subshells. The $3 d_{5 / 2}$ photoelectron peak is also seen on Fig 5 and 6 . The $3 d_{3 / 2}$ photoelectron peak is shifted on these figures into the region of the discrete spectrum: 
on Fig $5(\Delta E=0.2 \mathrm{eV})$ we can see only the sharp right wing of this distribution, on Fig $6(\Delta E=-0.3 \mathrm{eV})$ no trace of this peak is seen. It is seen that the less is the excess energy $\Delta E$ the more is the PCI distortion of the line shapes in full accordance with general PCI theory.

Then we can see on Fig 4 a weak structure between 0 and $1 \mathrm{eV}$ for the ${ }^{3} P$ state and a barely distinguishable structure near $1 \mathrm{eV}$ for the ${ }^{1} D$ state. These structures are associated with the decay of some intermediate resonant states. Because of the negligible probability of the PCI electron capture for this excess energy $\Delta E$ we conclude that this structure is due to the process of outer shell ionization (4). Note that the emission of the electrons in the ${ }^{3} P$ final state channel via VMD of the intermediate ionic state is stronger than in the ${ }^{1} D$ final state channel.

Much more pronounced structures can be seen on Fig 5 and 6 for the ${ }^{3} P$ and ${ }^{1} D$ final ionic states. These peaks are associated with the resonant processes (2) (for $\Delta E=0.2 \mathrm{eV}$ ) and (3) (for $\Delta E=-0.3 \mathrm{eV}$ ). The spectrum of these peaks is formed by the integrals $I$ (see equation (9)). The intermediate electron states both of the continuum and the discrete spectrum contribute to the integral $I$. But for each peak the main contribution comes only from a few discrete states (Sheinerman et al 2007).

The location of these peaks is determined by the structure of the discrete spectrum of the excited ion $K r^{+*}$, i.e. by the energies of the $4 p^{-2}\left({ }^{1} D,{ }^{1} S\right) n p$ and $4 p^{-2}\left({ }^{1} D,{ }^{1} S\right) n f$ states. The intensities of the peaks are determined by a few factors. First of all they depend on the matrix elements of the valence multiplet decay. Secondly, they depend on the probabilities of populating the excited ion $K r^{+*}$ states which differ in processes (2) and (3). In process (2) the population of the excited states occurs via the capture of the slow photoelectron. In reaction (3) - through the decay of the excited resonant states. The probability of such processes depends strongly on the excess energy above the threshold. The less is the excess photon energy $\Delta E$ the higher is the probability of population. Note that process (4) contributes also to the cross section. The population of the intermediate states in this process occurs due to the shake phenomenon (in our model). However for energies near threshold, $-1 \mathrm{eV}<\Delta E<0.5 \mathrm{eV}$, the population of the intermediate discrete states (especially, $4 p^{-2}\left({ }^{1} S\right) n p, n f$ states) by process (4) is notably less than by processes (2) and (3). Hence the main contribution to the cross section of the two electron emission in this region comes from the processes (1) and (2) of inner shell ionization .

Note that the contributions of processes (1) and (2) (or (3)) are comparable in magnitudes in the case of small excess energy $\Delta E$. Hence some interference effects might be expected in this case. Calculation taking into account the coherent sum of the amplitudes (solid lines) and incoherent contribution of the processes (dashed lines and color filling of the area under the curve) shows no notable difference for the case of the ${ }^{3} P$ final state. However, for the ${ }^{1} D$ final ionic state channel, there is some difference between the two calculations. The shapes of resonant lines on the left wing of the $3 d_{5 / 2}$ peaks reveal a remarkable contribution of the interference terms. 


\subsection{Comparison of calculated and measured spectra}

In order to compare the theoretical curves with the experimental data we have convoluted the calculated cross sections with a Gaussian function with a half width of $20 \mathrm{meV}$ (for small electron energy, $E_{k} \leq 3 \mathrm{eV}$ ) and of $80 \mathrm{meV}$ (for larger electron energy, $E_{k}>3 \mathrm{eV}$ ) to simulate the electron detector resolution. Convoluted curves are presented in Fig $7-9$ by solid lines together with the measured spectra. At each photon energy, the theoretical curves have been normalized to the experimental ones on the maximum of the $3 d_{5 / 2}$ peak in the ${ }^{1} S$ final state channel. Because HF calculations fail to reproduce the correct branching ratios (Sheinerman et al 2006) we have used the experimental values for this normalization. The normalization factor of the theoretical curves in the ${ }^{1} \mathrm{D}$ and ${ }^{3} \mathrm{P}$ channels is then obtained by correcting the calculated branching ratios which are approximately independent of the photon energy, $R\left({ }^{1} D:{ }^{1} S\right)=1.35$ and $R\left({ }^{3} P:{ }^{1} S\right)=2.15$, to the experimental ones which are respectively 1.59 and 1.27 , as deduced from our measurement in the $3 d_{5 / 2}$ channel (Fig 7 ). Fig $7-9$ show then a reasonable agreement between the calculated and measured cross sections.

In Fig 7, the two large peaks show that the main process for two electron emission at $\Delta E=5.0 \mathrm{eV}$ is, in all channels, the $3 \mathrm{~d}$ ionization distorted by PCI effects, process (1). In the ${ }^{3} P$ channel the relative intensity of these peaks is very well reproduced by our calculation. However in the ${ }^{1} D$ and ${ }^{1} S$ channels the measured intensity of the $3 d_{3 / 2}$ peak exceeds notably the calculated one. Note that the same discrepancy was observed in the intensities of the threshold electron spectra in Xe (Sheinerman et al 2006). This indicates that the HF calculation of the matrix elements of the Auger decay with LScoupling is not sufficient to describe correctly the relative strength of the electron lines.

In Fig 7 (c), both experiment and calculation show structure on the spectrum of the ${ }^{3} P$ final state in the region of small electron energies, $E<2 \mathrm{eV}$, originating from the outer shell ionization followed by VMD, eq. (4). However the experimental structure is more pronounced and extended compared to the calculated one. A reason for this discrepancy lies probably in the limitations of theoretical model used. Population of the $K r^{*+}\left(4 p^{-2}\right)\left({ }^{1} D\right) n d\left({ }^{2} S\right)$ Rydberg series, which are dominant according to Alitalo et al (2001) is for instance not included in our model. Our approach considers only the shake process for the excitation of a second electron of the outer shell and neglects other mechanisms which can play an important role (Chang and Poe 1975, Carter and Kelly 1977). Taking into account the correlation in the initial state and the knocking out of the second electron by the first one we can increase the intensity of the resonance lines. But such elaborate calculations are beyond our model.

Our calculations reproduce well the PCI distorted line shape of the $3 d_{5 / 2}$ electrons measured in the ${ }^{1} S$ channel also for smaller photon energies $\omega$ (Fig 8 and 9 (a)), and we observe that the PCI distorted 3d ionization (Eq.1) is the dominant process that leads to two electron emission in this channel for all photon energies considered. On the other hand in the ${ }^{1} D$ and ${ }^{3} P$ channels the processes $(2)-(4)$ contribute to the cross section in the region of small electron energies. They are revealed in Fig 8 and 
9 (c) as a dense structure in the region $E<2 \mathrm{eV}$. The calculated curves describe quite well the contribution from the PCI distorted line shapes of the photoelectrons. However the intensities and location of the calculated and measured resonance lines differ. It was noted in the previous section that the location of these peaks is determined by the structure of the discrete spectrum of the excited ion $K r^{+*}$, which have been considered in the HF approximation. The intensities of the peaks are determined by the matrix elements which have been also calculated in the HF approximation. Hence the disagreement between the calculated and measured resonance lines shows that the nonrelativistic HF approximation is not sufficient to describe precisely the peaks associated with the processes (2)-(4). It is possible that more realistic approximations like $j l$ coupling for the description of the atomic levels (Sobelman 1992) or taking into account many electron correlation for the valence multiplet decay could shift the position and the strength of the calculated peaks. Such calculation is time consuming and lies beyond the framework of this paper.

Let's analyze now the resonance structure in the $4 p^{-2}\left({ }^{3} P\right)$ channel for different photon energies $\omega$ (Fig 7c, 8c, 9c and Fig 3). For the energies $\omega=100.04,98.54,85 \mathrm{eV}$ which are relatively far from threshold the probabilities of the electron recapture or of the resonance Auger decay (equations (2), (3)) are quite small. Hence a notable contribution to the measured spectra comes from the process of outer shell ionization, equation (4), which occurs through the excitation of the $4 p^{-2}\left({ }^{1} D\right) n l$ intermediate states. However, the similarity of the structure at all the presented energies shows that process (4) contributes also significantly for the energies $\omega=95.24,94.74 \mathrm{eV}$, where we could expect the dominant role of the processes (2), (3). Our calculation underestimates the contribution of the process (4). Hence the calculated structure on the Fig $8 \mathrm{c}, 9 \mathrm{c}$ is explained by the dominant contribution of the processes (2), (3). On the other hand in the channel of the $4 p^{-2}\left({ }^{1} D\right)$ final state both the measurement and calculation show a little role for the process (4). So the structure on the Fig $8 \mathrm{~b}, 9 \mathrm{~b}$ is solely due to the contribution of the processes (2), (3) which are going on through the creation and decay of the $4 p^{-2}\left({ }^{1} S\right) n p, n f$ intermediate states.

Another point needs discussion: the two electron emission yield in Fig 8 b,c in the region $1.8<E<2.3 \mathrm{eV}$ for the ${ }^{1} D$ final ionic state, and in the region $1.4<E<1.8 \mathrm{eV}$ for the ${ }^{3} P$ state. At this photon energy, a strong PCI capture of the $3 d_{3 / 2}$ photoelectron (process (2)) occurs, and these electrons are the autoionizing electrons produced upon the VMD of the Rydberg states with $4 p^{-2}\left({ }^{1} S\right)$ and $4 p^{-2}\left({ }^{1} D\right)$ ionic cores, respectively. The energy and distributions of these electrons reflect the probability of the PCI capture into the different intermediate Rydberg states. The quasi continuum distributions show that recapture is predominantly to Rydberg states of high quantum number $n$. Because of the limited number of the discrete states $\left(n_{\max }=15\right)$ included in our calculation, we cannot reproduce this distribution.

We turn now to Fig 9, and to the Rydberg structure we observe in channels ${ }^{1} D$ and ${ }^{3} P$, associated with the resonant Auger process (Eq.(3)). The photon energy of $94.74 \mathrm{eV}, 300 \mathrm{meV}$ below the $3 d_{3 / 2}$ threshold corresponds roughly to a $3 d_{3 / 2} \rightarrow 9 p$ 
excitation. The experimental data in the ${ }^{1} D$ channel, Fig $9 \mathrm{~b}$ shows that the resonant Auger decay (Eq.(3)) populates mainly the $4 p^{-2}\left({ }^{1} S\right) n p$ Rydberg states with $\mathrm{n}=11,10$ and 9 and more weakly the ones with $\mathrm{n}=12$ and 8 . Weak population of other $4 p^{-2}\left({ }^{1} S\right) n l$ Rydberg series can also be noticed. This is in very good quantitative agreement with the observation of Aksela et al (Aksela 1997) upon $3 d_{5 / 2} \rightarrow 9 p$ excitation. The calculated spectrum in Fig 9b reproduces qualitatively the main experimental features: intensities differ slightly due to the limitations of the HF model described above, but the position of the Rydberg series is well predicted. For ${ }^{3} P$ channel, Fig $9 \mathrm{c}$, peaks correspond to the autoionization of $4 p^{-2}\left({ }^{1} D\right) n p$ Rydberg series in the $K r^{2+} 4 p^{-2}\left({ }^{3} P\right)$ channel, and the experiment resolves here autoionization to the three $\left({ }^{3} P_{2,1,0}\right)$ components of the $K r^{2+}$ final state, as demonstrated by the assignment reported on the Fig 9c. As expected, the relative experimental intensities of the different $n$ members of a Rydberg series are similar to that of the $4 p^{-2}\left({ }^{1} S\right) n p$ series in Fig 9b. As the HF model considers only one single $K r^{2+} 4 p^{-2}\left({ }^{3} P\right)$ state, only one series of autoionizing lines is predicted. Calculation predicts that autoionization of the $K r^{2+} 4 p^{-2}\left({ }^{1} D\right) 11 p$ and $K r^{2+} 4 p^{-2}\left({ }^{1} D\right) 9 f$ Rydberg states gives a peak at $0.51 \mathrm{eV}$. This position matches rather the experimental value for the autoionization to the $K r^{2+} 4 p^{-2}\left({ }^{3} P_{1}\right)$ final state.

Finally, it should be noted that the interference effects which are predicted on the left wings of the calculated $3 d_{5 / 2}$ line shape cannot be clearly confirmed by the experimental data because the resolution of our measurements is not sufficient to observe these effects, and also because the resonant contribution seems smaller than predicted, in the experimental spectra for the ${ }^{1} D$ final state.

\section{Conclusion}

We have considered the double ionization of Krypton by photon impact when the energy of the incident photons is close to the threshold of the $3 d$ inner shell. The method of electron - electron coincidences has allowed us to measure the yield of DPI in the channels with the precisely selected final ionic state $4 p^{-2}\left({ }^{2 S+1} L\right)$. The analysis of the measured spectra which is based on a quantum mechanical calculation has revealed three processes leading to the emission of two electrons in the considered region. They are: 1) the $3 d$-shell ionization followed by the Auger decay, distorted by PCI; 2) the PCI recapture of the slow $3 d$ photoelectron into a discrete state followed by VMD; 3 ) the outer $4 p$-shell ionization with simultaneous excitation of another $4 p$ electron followed by VMD. The calculated cross sections agree reasonably with the measured spectra, and reproduce the main trends. The analysis of the calculated and measured spectra shows the role of the different processes for the two electron emission. In the ${ }^{1} S$ final ionic state channel the DPI yield is formed solely by the ionization of the $3 d$ shell followed by the Auger decay. In the ${ }^{1} D$ and ${ }^{3} P$ channels all three processes play an important role. The processes which involve the creation of an intermediate state followed by VMD contribute in the region of small electron energy $E<2 \mathrm{eV}$ whereas the direct $3 d-$ ionization is observed in different regions, depending on the photon energy. For small 
excess energy above threshold, $\Delta E<0.5 \mathrm{eV}$, the contributions of all the processes are comparable in magnitude and lead to pronounced structures in the spectra.

Both the experimental method and the theoretical approach presented in this paper can be applied also to the investigation of the other inner shells and other atoms. The combination of our measured and calculated data shows clearly that present techniques allow us to study different processes involved in the decay dynamics of inner-shell excited systems. At the same time, they also show that much more elaborate efforts are necessary to obtain a proper model for all details of the involved many-body effects.

\section{Acknowledgments}

S.S is grateful to the LCP-MR, Université P. and M. Curie and CNRS for financial support. The project was approved by the Bessy committee program and was supported in part by the European Community, Research Infrastructure Action under the FP6 Structuring European research Area Programme (contract R II 3-CT-2004-506008).

\section{References}

Electronic address: penent@ccr.jussieu.fr

Aksela H, Kivilompolo M, Nõmmiste E and Aksela S 1997 Phys. Rev. Lett. 794970

Alitalo S, Kivimäki A, Matila T, Vaarala K, Aksela H and Aksela S 2001 J. Electron Spectrosc. Related Phenom. 114-116 141-146

Amusia M Ya 1990 Atomic Photoeffect (Plenum Press, New York and London)

Armen G B, Aksela H, Ảberg T and Aksela S 2000 J. Phys. B: At. Mol. Opt. Phys. 33 R49

Armen G B and Larkins F P 1991 J. Phys. B: At. Mol. Opt. Phys. 24 741-59

Armen G B and Larkins F P 1992 J. Phys. B: At. Mol. Opt. Phys. 25 931-47

Avaldi L and Huetz A 2005 J. Phys. B: At. Mol. Opt. Phys. 38 S861

Becker U, Wehlitz R, Hemmers 0, Langer B and Menzel A 1989 Phys. Rev. Lett. 631054

Becker U, Hemmers 0, Langer B, Lee I, Menzel A and Wehlitz R 1993 Phys. Rev. A 47 R767

Briggs J S and Schmidt V 2000 J. Phys. B: At. Mol. Opt. Phys. 33 R1

Carter S L and Kelly H P 1977 Phys. Rev. A 16 1525-34

Chang T N and Poe R T 1975 Phys. Rev. A 12 1432-39

Eland J H D, Vieuxmaire O, Kinugawa T, Lablanquie P, Hall R I and Penent F 2003 Phys. Rev. Lett. 90053003

Feng X, Wills A A, Sokell E, Gorczyca T W, Wiedenhoeft M and Berrah N 2005 Phys. Rev. A 72 042712

Hikosaka Y, Penent F, Lablanquie P, Hall R I and Ito K 2000 Meas. Sci. Technol. 111697

Jurvansuu M, Kivimäki A, and Aksela S 2001 Phys. Rev. A 64012502

Kitajima M, Yoshida H, De Fanis A, Prümper, Hergenhahn U, Kukk E, Tanaka T, Nakagawa K, Tanaka H, Fritzsche S, Sazhina I P, Kabachnik N M and Ueda K 2006 J. Phys. B: At. Mol. Opt. Phys. 39 1299

Kuchiev M Yu and Sheinerman S A 1985 J. Phys. B: At. Mol. Phys. 18 L551

Kuchiev M Yu and Sheinerman S A 1986 Comp. Phys. Comm. 39155

Kuchiev M Yu and Sheinerman S A 1989 Sov. Phys.-Usp. 32569

Lablanquie P, Sheinerman S, Penent F, Aoto T, Hikosaka Y and Ito K, 2005 J. Phys. B: At. Mol. Opt. Phys. 38 L9

Lablanquie P, Andric L, Palaudoux J, Becker U, Braune M, Viefhaus J, Eland J, and Penent F, 2007 J. Electron Spectrosc. Related Phenom. 156-158 51 
National Institute of Standards and Technology (NIST), Atomic Spectra Database Energy Levels, 2007, Data available online at http://physics.nist.gov/PhysRefData/ASD/

Penent F, Palaudoux J, Lablanquie P, Andric L, Feifel R and Eland J H D, 2005 Phys. Rev. Lett. 95 083002

Penent F, Lablanquie P, Hall R I, Palaudoux J, Ito K, Hikosaka Y, Aoto T and Eland 2005A J. Electron Spectrosc. Related Phenom. 144-147 7

Schmidt V, 1992 Rep. Prog. Phys. 55, 1483

Schmidt V, 1997 Electron Spectrometry of Atoms using Synchrotron Radiation, (University Press, Cambridge, 1997), pp.275,276

Sheinerman S, 2003 J. Phys. B: At. Mol. Opt. Phys. 364435

Sheinerman S, Lablanquie P, Penent P, Palaudoux J, Eland J H D, Aoto T, Hikosaka Y and Ito K 2006 J. Phys. B: At. Mol. Opt. Phys. 39 1017-33

Sheinerman S, Lablanquie P and Penent F 2007 J. Phys. B: At. Mol. Opt. Phys. 40 1889-1906

Sobelman I I 1992 Atomic Spectra and Radiative Transitions, (Springer-Verlag, Berlin, 1992), pp.46-48 


\section{Figure Captions}

Fig 1: Sketch of the different processes leading to two electron emission, as described in paragraph 2. Three ionization channels $K r^{+} 3 d^{-1}, K r^{2+} 4 p^{-2}\left({ }^{2 S+1} L\right)$ and $K r^{2+} 4 p^{-2}\left({ }^{2 S_{1}+1} L_{1}\right)$ are represented, with the continuum part (such as $K r^{+} 3 d^{-1}+e$ ) schematized by a rectangle and the associated Rydberg series (such as $K r 3 d^{-1} n p$ ) schematized by horizontal bars. (1) is 3d ionization with Auger decay, affected by PCI. (2) is 3d recapture followed by VMD. (3) Resonant Auger with VMD.(4) is indirect valence DPI.

Fig 2: (color on line) Kr Double Photoionization brought about by $95.24 \mathrm{eV}$ photons, 0.2 $\mathrm{eV}$ above the $3 d_{3 / 2}$ threshold. Top panel displays the two dimensional electron / electron coincidence map. Intensity is plotted on a linear scale. Diagonal lines of constant kinetic energy sum correspond to the different $K r^{2+}$ final states. Bottom panel gives the DPI continua of three selected such states. They have been obtained from the coincidence map by projecting intensity of the corresponding diagonal lines on the $\mathrm{y}$, slow electron energy axis. Background estimated off the diagonal has been subtracted. The Kr $3 d_{5 / 2}$ photoelectron asymmetric peak is observed at $1.35 \mathrm{eV}$. Assignment is given for the VMD of the Rydberg states into which the $3 d_{3 / 2}$ photoelectron has been captured.

Fig 3: Dependance of the $K r^{2+}\left(4 p^{-2}\right){ }^{3} P_{2}$ (bottom spectra in red) and ${ }^{1} D$ (top spectra in black) DPI continua on the excitation energy. Left panel shows the spectra obtained at $85 \mathrm{eV}$, below the $3 \mathrm{~d}$ excitation threshold. Fig 3 Middle panel was measured $3.5 \mathrm{eV}$ above the $\mathrm{Kr} 3 d_{3 / 2}$ threshold and right panel $0.2 \mathrm{eV}$ above. Intensities correspond to the measured coincidence counts and can be directly compared when corresponding to a same photon energy. Contribution of false coincidences has been removed.

Fig 4: Electron energy distributions for DPI into the $K r^{2+}{ }^{1} S,{ }^{1} D$ and ${ }^{3} P$ continua calculated at a photon energy $\omega=100.04 \mathrm{eV}, 5 \mathrm{eV}$ above the $3 d_{3 / 2}$ threshold.

Fig 5: Same as in Fig 4, but for a photon energy $200 \mathrm{meV}$ above the $3 d_{3 / 2}$ threshold. The solid lines results from the coherent contribution of the different processes, and the dashed line to the incoherent one. The shaded area corresponds to the contribution of the $3 \mathrm{~d}$ photoionization only (process $(1)$ ) .

Fig 6: Same as in Fig 4, but for a photon energy $300 \mathrm{meV}$ below the $3 d_{3 / 2}$ threshold.

Fig 7: Measured $K r^{2+}$ DPI continua observed with a photon energy $5 \mathrm{eV}$ above the $3 d_{3 / 2}$ threshold and compared with the calculated DPI cross section from Fig 4. The electron count rate was 10000 per second and the accumulation time $10 \mathrm{~min}$. The histograms correspond to a discretization with $10 \mathrm{meV}$ steps.

Fig 8: Same as in Fig 7, but for a photon energy $200 \mathrm{meV}$ above the $3 d_{3 / 2}$ threshold.

Fig 9: Same as in Fig 7, but for a photon energy $\omega=94.74 \mathrm{eV}, 300 \mathrm{meV}$ below the $3 d_{3 / 2}$ threshold, and $40 \mathrm{meV}$ above the $3 d_{3 / 2} \rightarrow 9 p$ excitation at $94.70 \mathrm{eV}$. The assignment of the Rydberg series populated by the resonant Auger decay (process (3)) is indicated. 


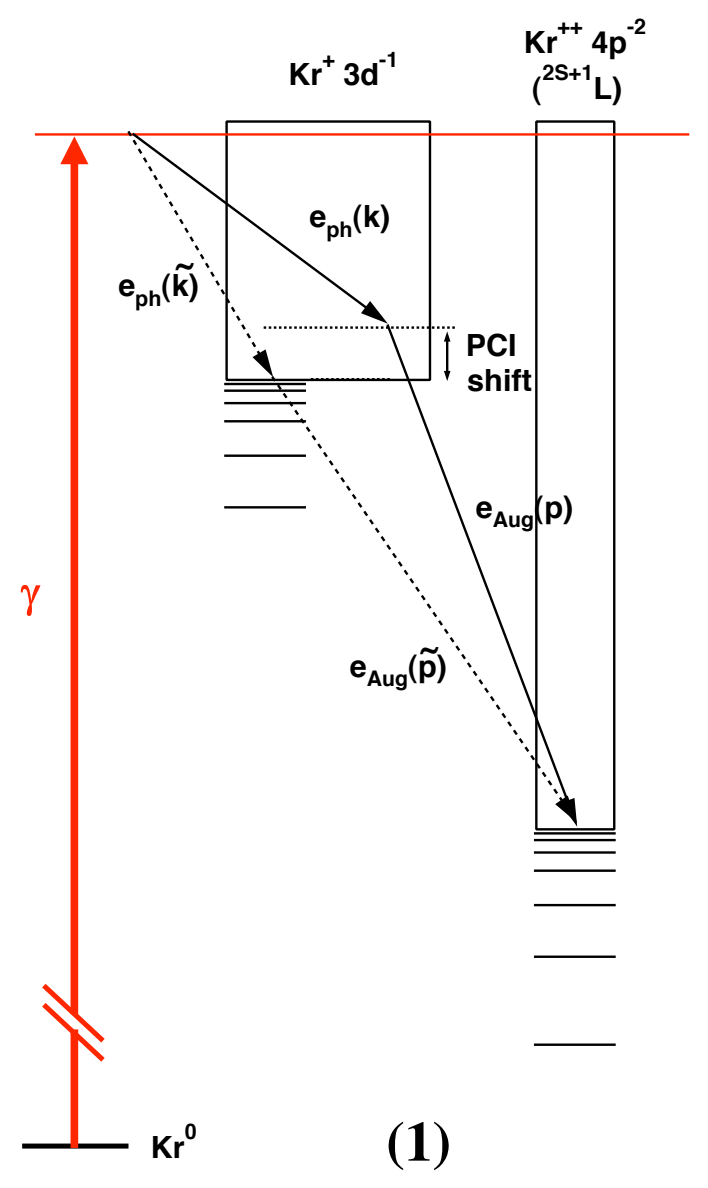

3d Ionization \& PCI

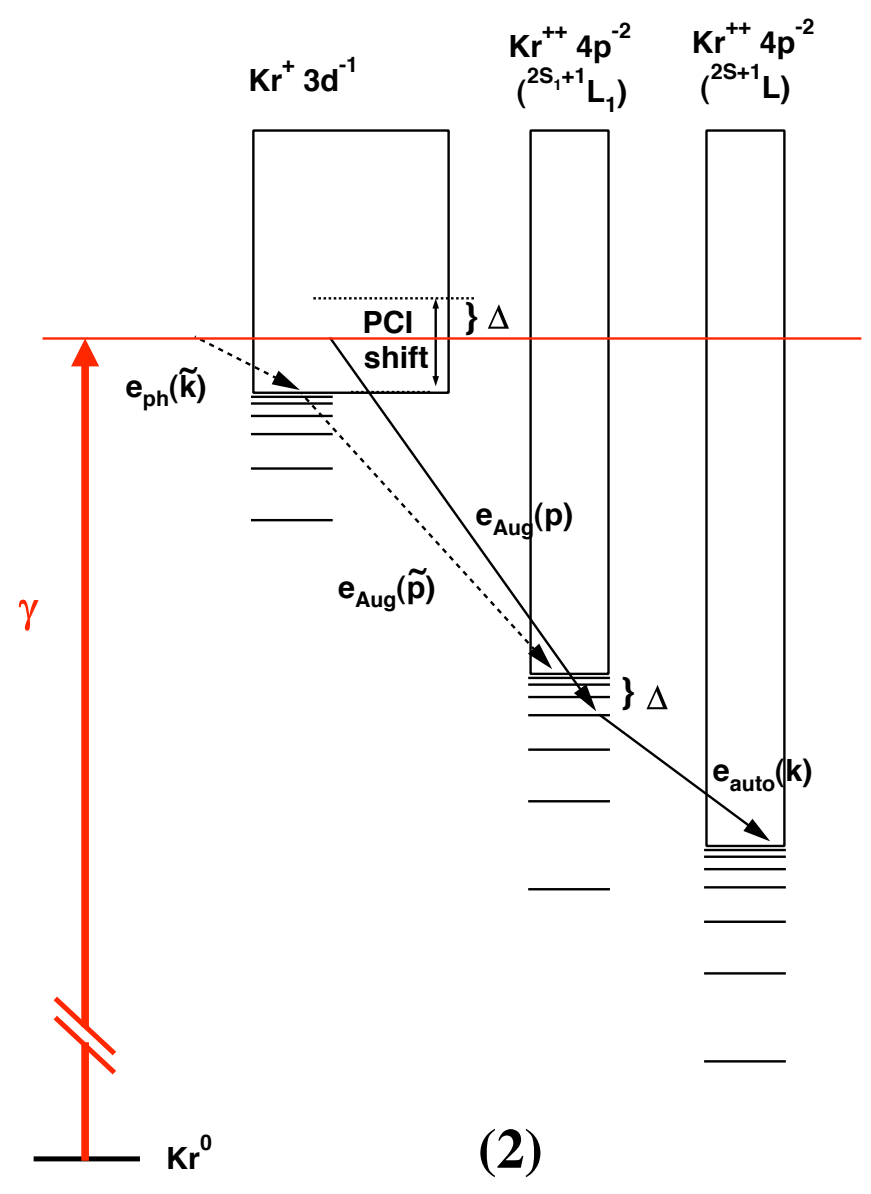

3d Recapture \& VMD

Figure 1a (Fig1 A.eps) 


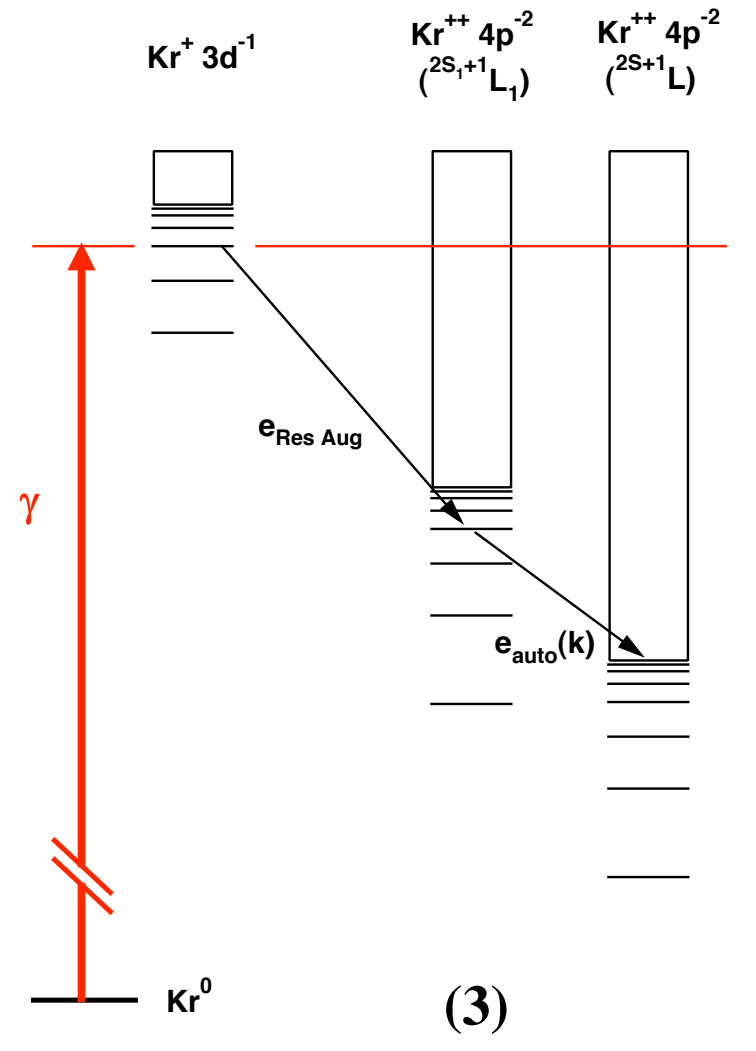

Resonant Auger \& VMD

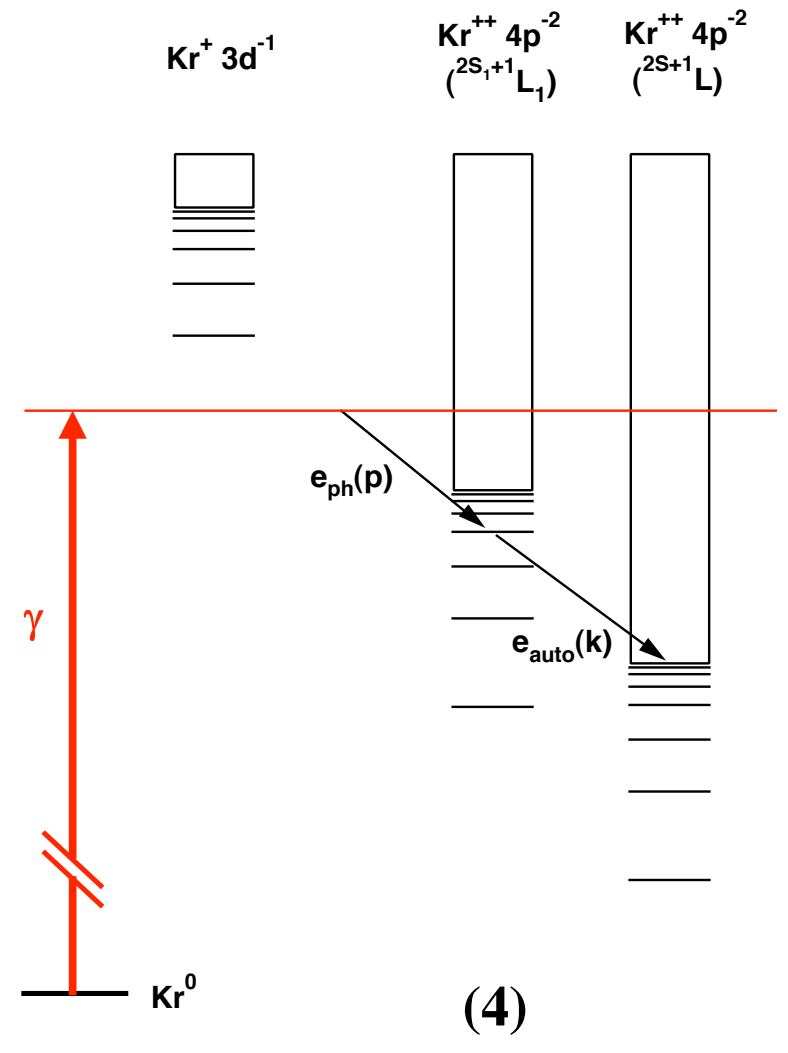

valence DPI \& VMD

Figure 1b (Fig1 B.eps) 

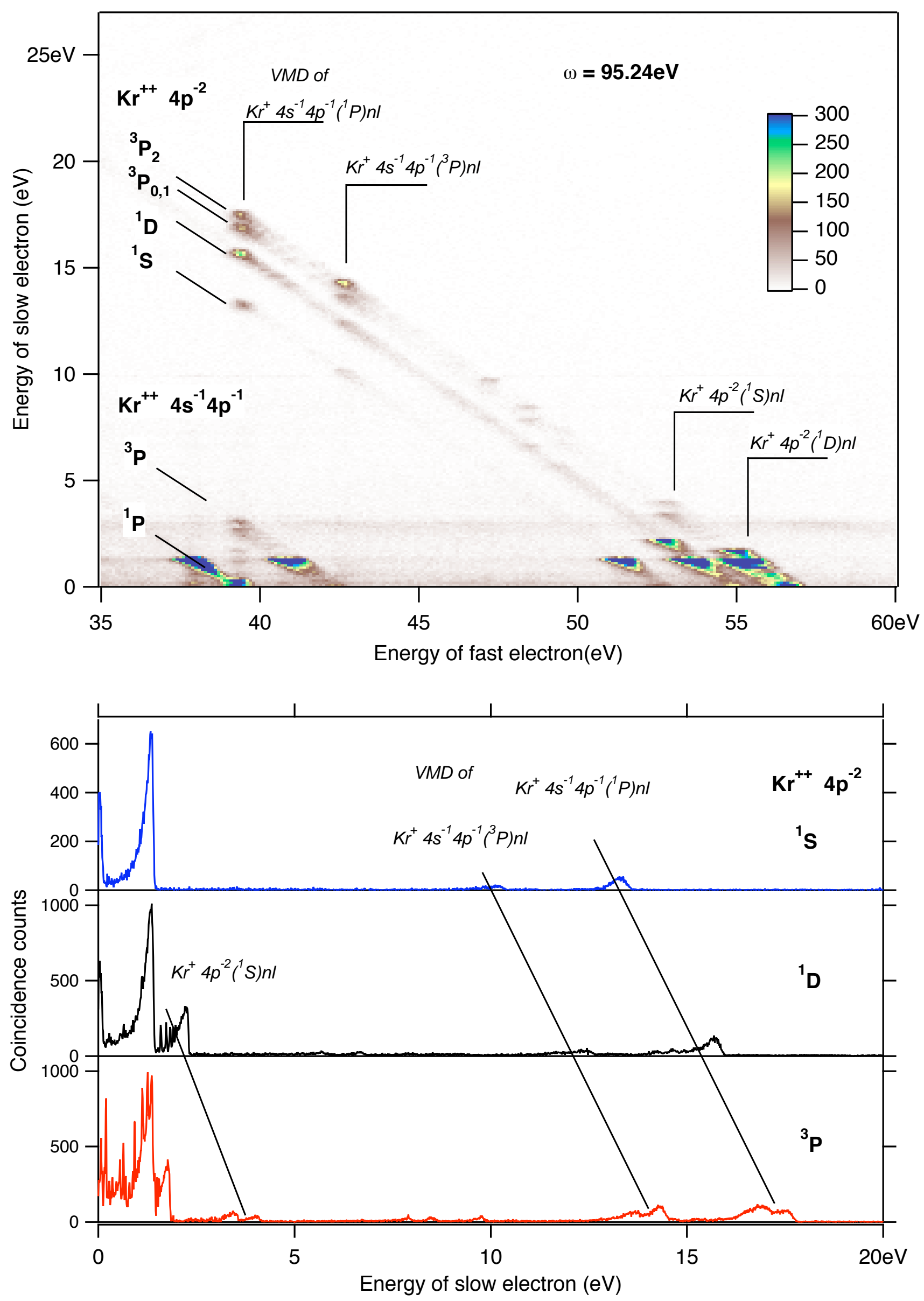

Figure 2 (Fig 2.eps) 

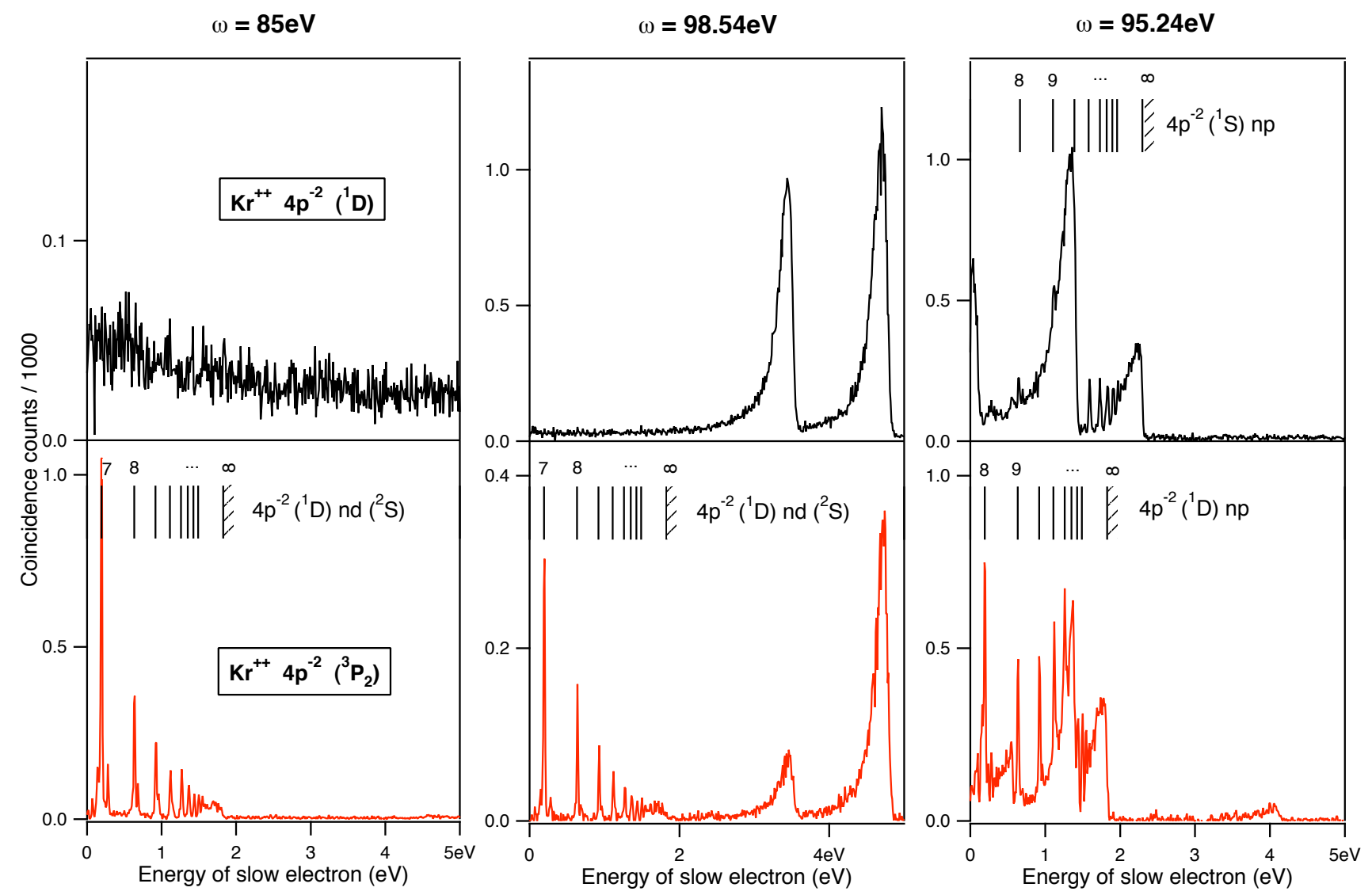

Figure 3 (Fig 3.eps) 


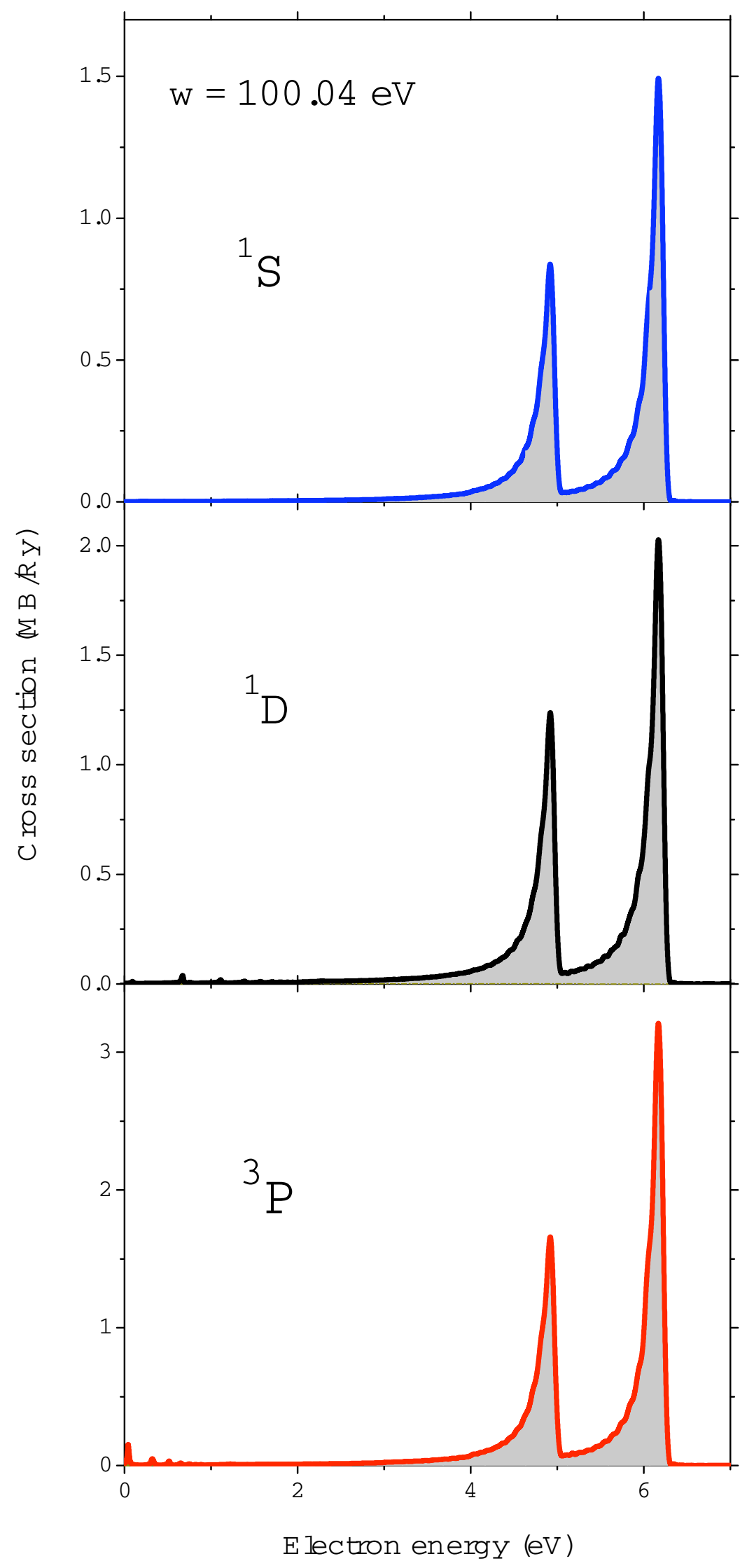

Figure 4 (Fig 4.eps) 


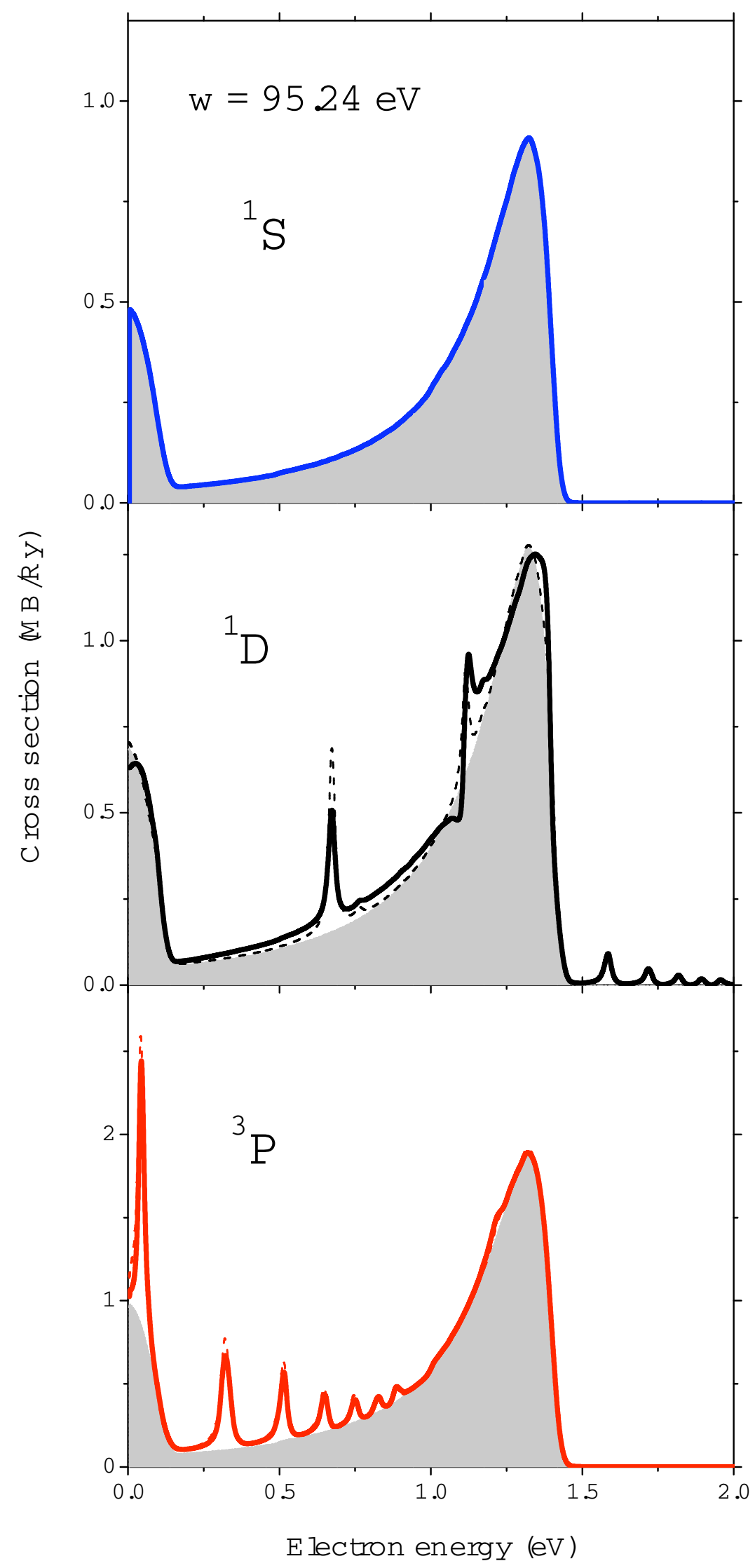

Figure 5 (Fig 5.eps) 


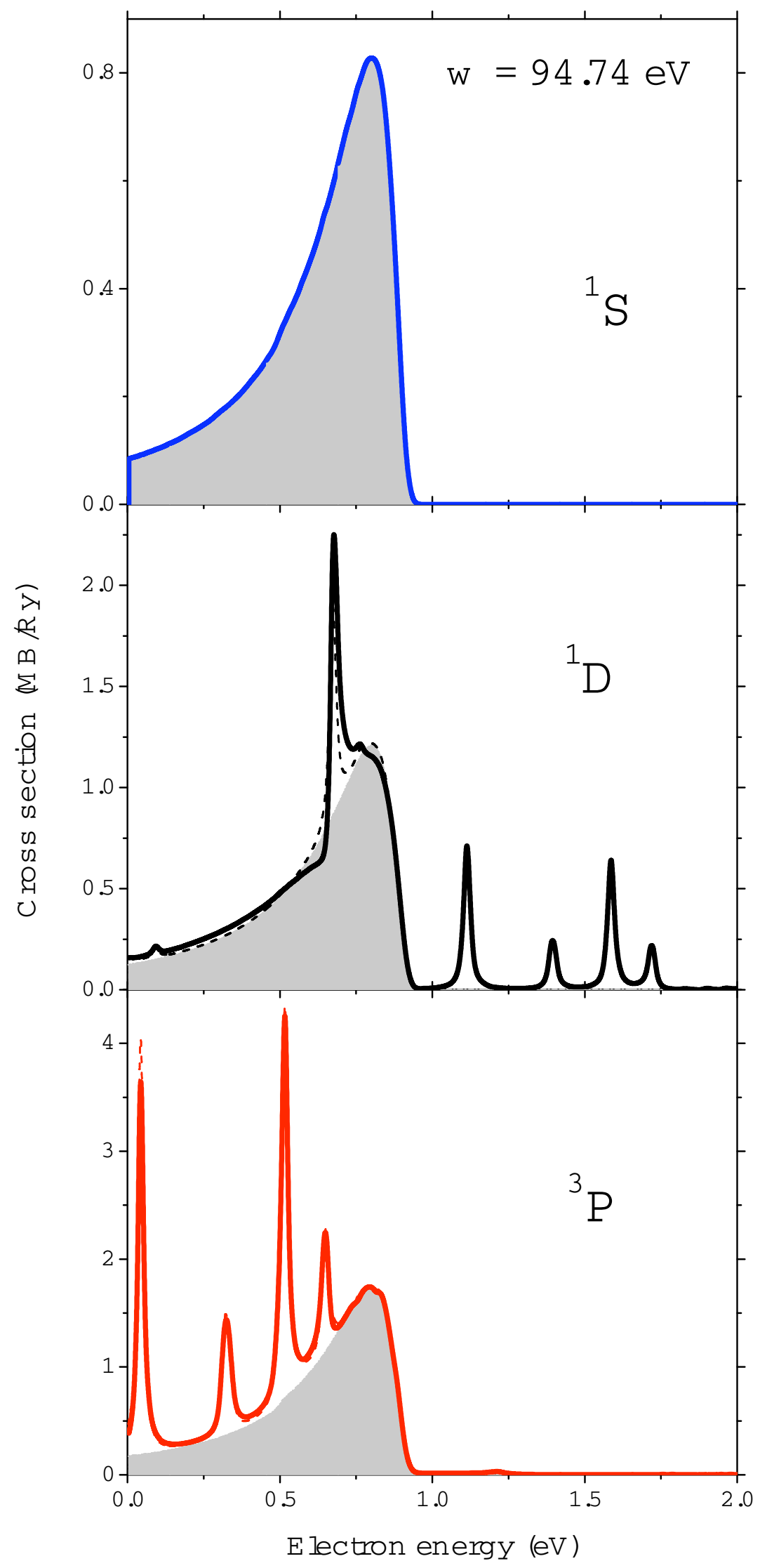

Figure 6 (Fig 6.eps) 


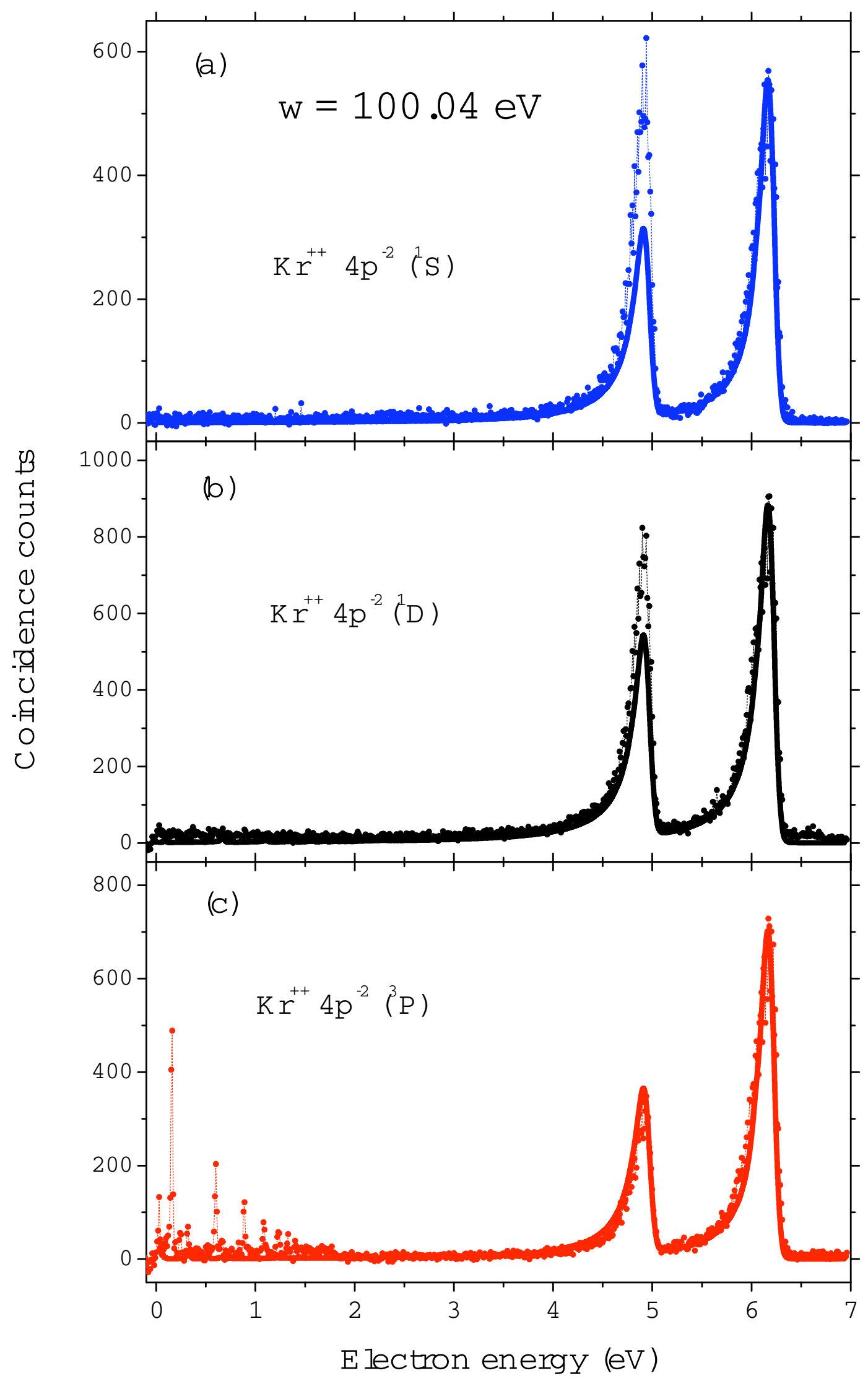

Figure 7 (Fig 7.eps) 


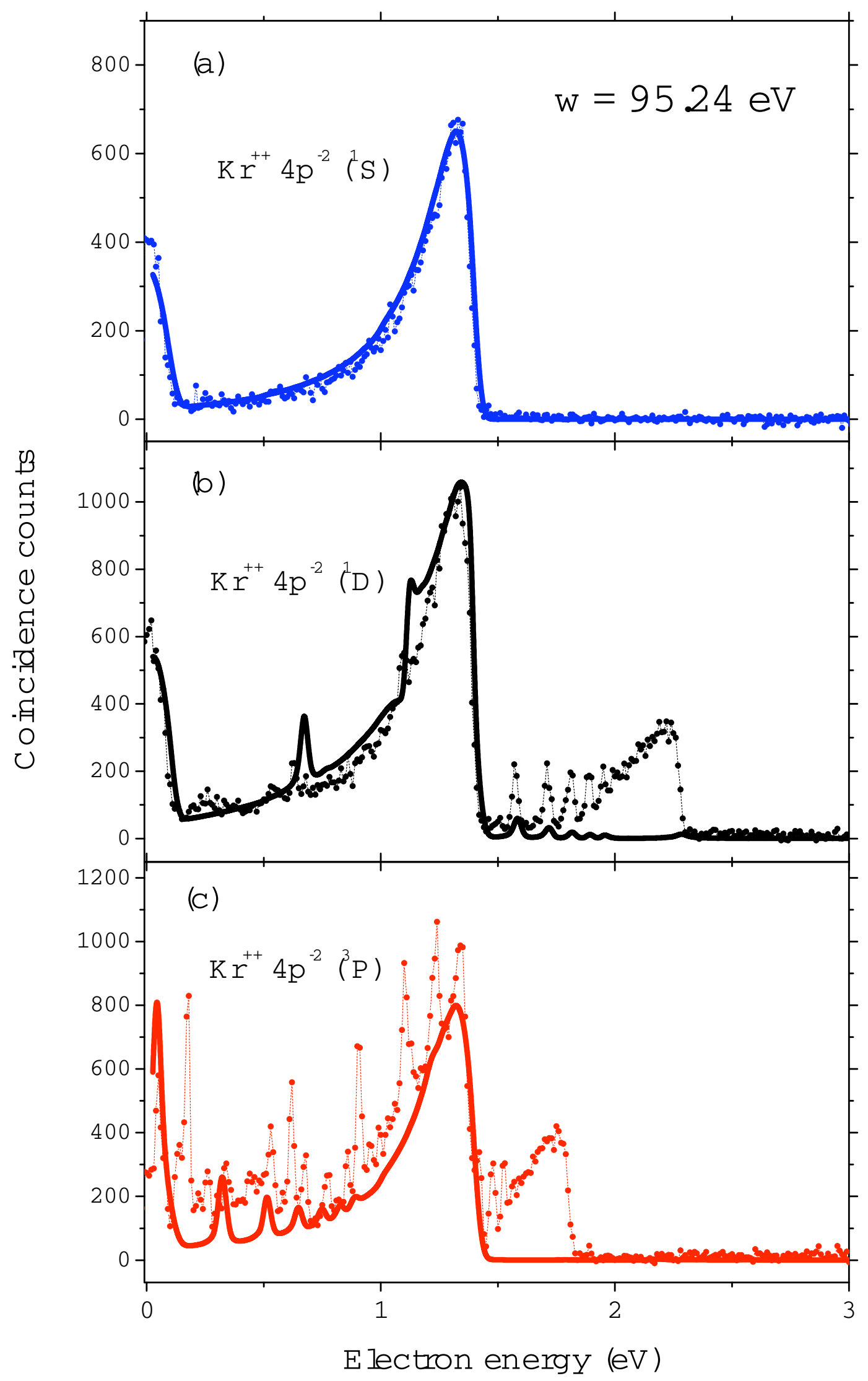

Figure 8 (Fig 8.eps) 


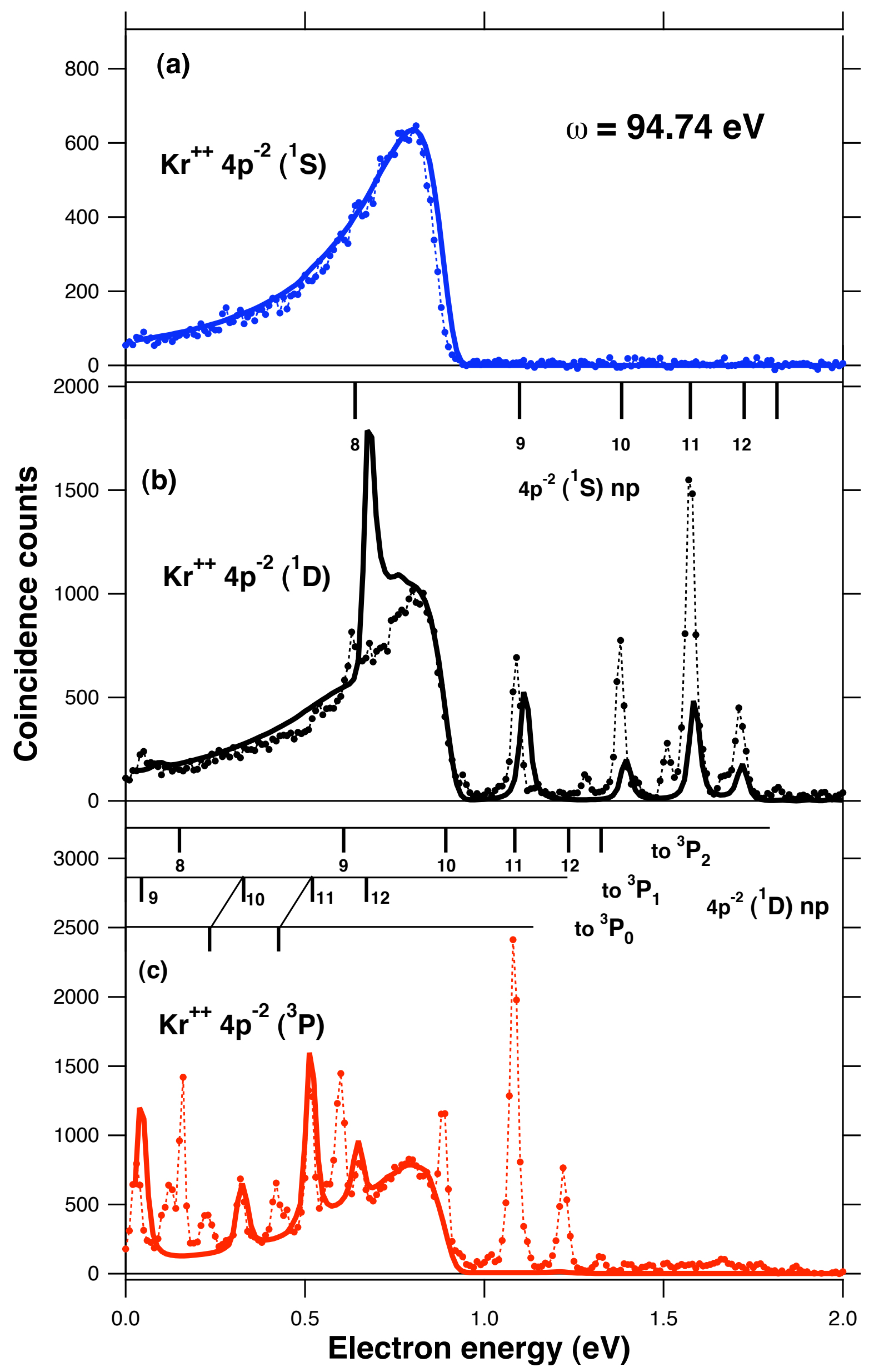

Figure 9 (Fig 9.eps) 